OUTP-00-41P (rev)

\title{
Low-scale inflation
}

\author{
Gabriel Germán \\ Centro de Ciencias Físicas, Universidad Nacional Autónoma de México, Apartado Postal 48-3, \\ 62251 Cuernavaca, Morelos, México \\ Graham Ross and Subir Sarkar \\ Theoretical Physics, University of Oxford, 1 Keble Road, Oxford OX1 3NP, UK
}

(October 31, 2018)

\begin{abstract}
We show that the scale of the inflationary potential may be the electroweak scale or even lower, while still generating an acceptable spectrum of primordial density perturbations. Thermal effects readily lead to the initial conditions necessary for low scale inflation to occur, and even the moduli problem can be evaded if there is such an inflationary period. We discuss how low scale inflationary models may arise in supersymmetric theories or in theories with large new space dimensions.
\end{abstract}

98.80.Cq, 04.65.+e, 98.65.Dx, 98.70.Vc 


\section{INTRODUCTION}

Inflation provides an attractive context for discussion of the initial conditions of the hot big bang cosmology, as well as a very plausible mechanism for generation of the scalar density perturbations which have left their imprint in the anisotropy of the cosmic microwave background (CMB) and grown into the observed large-scale structure (LSS) of galaxies [1,2]. Field theoretical models of inflation are typically of the 'slow-roll' type in which an inflaton field, $\phi^{*}$, evolves along a quasi-flat potential to its global minimum. During the roll the vacuum energy is approximately constant and drives an era of exponential growth in the cosmological scale factor $a$. However such field theory models must explain why the potential is extremely flat even in the presence of radiative corrections — the " $\eta$-problem" — and also explain why the inflaton field initially lies far from its true minimum.

Quite generally, we may expand the inflaton potential about the value of the field $\phi_{\mathrm{I}}^{*}$ just before the start of the observable inflation era when the scalar density perturbation on the scale of our present Hubble radius 1 was generated, and expand in the field $\phi \equiv \phi^{*}-\phi_{\mathrm{I}}^{*}$ [3]. Since the potential must be very flat to drive inflation, $\phi$ will necessarily be small while the observable density perturbations are produced, so the Taylor expansion of the potential will be dominated by low powers:

$$
V(\phi)=V(0)+V^{\prime}(0) \phi+\frac{1}{2} V^{\prime \prime}(0) \phi^{2}+\ldots
$$

The first term $V(0)$ provides the near-constant vacuum energy driving inflation while the $\phi$ dependent terms are ultimately responsible for ending inflation, driving $\phi$ large until higherorder terms violate the slow-roll conditions needed for inflation. These terms also determine the nature of the density perturbations produced, in particular the departure from an exactly scale-invariant spectrum.

The density perturbation at wavenumber $k$ is given by [2]

$$
\delta_{\mathrm{H}}^{2}(k)=\frac{1}{75 \pi^{2}} \frac{V\left(\phi^{*}=\phi_{\mathrm{H}}^{*}\right)^{3}}{V^{\prime}\left(\phi^{*}=\phi_{\mathrm{H}}^{*}\right)^{2} M^{6}}
$$

where $\phi_{\mathrm{H}}^{*}$ is the value of the inflaton field when the relevant scale 'exits the horizon', i.e. when $k=a H, H \equiv \dot{a} / a \simeq\left(V / 3 M^{2}\right)^{1 / 2}, M \equiv M_{\mathrm{P}} / \sqrt{8 \pi} \simeq 2.44 \times 10^{18} \mathrm{GeV}$. The slope of the potential is given by Eq.(1) as

$$
V^{\prime}\left(\phi^{*}=\phi_{\mathrm{H}}^{*}\right)=V^{\prime}(0)+V^{\prime \prime}(0) \phi_{\mathrm{H}}+\ldots,
$$

where $\phi_{\mathrm{H}} \equiv \phi_{\mathrm{H}}^{*}-\phi_{\mathrm{I}}^{*}$. If the term linear in $\phi$ in the potential (11) dominates when the observed density perturbations are generated ("linear inflation"), then only the first term above is important and the scale of the inflationary potential is required to be near the Planck scale. This can be seen by writing the slope as $V^{\prime}(0)=c V(0) / M$ which gives

\footnotetext{
${ }^{1}$ Numerically this is $H_{0}^{-1} \simeq 3000 h^{-1} \mathrm{Mpc}$, where $h \equiv H_{0} / 100 \mathrm{~km} \mathrm{~s}^{-1} \mathrm{Mpc}^{-1} \sim 0.65 \pm 0.15$ is the present Hubble parameter. LSS and CMB observations can measure the density perturbation down to galactic scales $(\sim 1 \mathrm{Mpc})$, a spatial range corresponding to just $7-8$ e-folds of inflation.
} 


$$
V^{1 / 4}(\phi=0) \simeq\left(75 \pi^{2} \delta_{\mathrm{H}}^{2}\right)^{1 / 4} c^{1 / 2} M \sim 2 \times 10^{-2} \sqrt{c} M,
$$

using the COBE determination of $\delta_{\mathrm{H}} \simeq 2 \times 10^{-5}$ on the scale of the present Hubble radius 四. We see that the inflationary scale is below the Planck scale (so a field-theoretic description may be used) but, unless $c$ is unnaturally small, will be not too far below it [1.2].

Examples of models generating linear inflation abound. Consider for example the commonly used 'chaotic inflation' models in which the inflationary potential is dominated by a monomial, $V \propto \phi^{* n}$, and $\phi_{\mathrm{I}}^{*}$ is larger than the Planck scale [0]. The terms in the Taylor expansion satisfy:

$$
V^{p+1}(\phi=0) \phi^{p} / V^{\prime}(\phi=0) \simeq\left(\phi / \phi_{\mathrm{I}}^{*}\right)^{p} \ll 1 .
$$

Consequently the linear term dominates the $\phi$ dependence showing that any such large-field inflation model with a monomial potential actually falls into the class of linear inflation models defined above. Hence the inflationary scale is not too far below the Planck scale, e.g. for the commonly adopted potential $V=\frac{1}{2} m^{2} \phi^{* 2}$ one requires $m \lesssim 10^{-4} M$.

There is a circumstance, however, in which it is possible in a natural way to avoid the conclusion that the inflationary scale is large. This is the case if the linear term is anomalously small. If, as is the usual expectation, the field $\phi^{*}$ transforms under some symmetry of the underlying theory, a linear term is forbidden if one expands the potential about the symmetric point. In this case the natural expectation is that the next term, namely the quadratic term will dominate. In the large-field inflation model just discussed, this situation does not arise because the initial value of the inflaton field corresponds to a (chaotic) value, and any symmetry under which it is charged is broken. However, as we shall discuss, it is possible that the initial conditions are such as to set the inflaton field close to the origin where the symmetry is unbroken, as in 'new inflation' [0]. Provided $\phi_{\mathrm{I}}^{*}$ is also close to the origin, the term linear in $\phi$ in the potential (四) will indeed be very small, so the quadratic term may dominate.

It is the purpose of this paper to investigate this possibility ("quadratic inflation") in detail. Following the same procedure as above we may determine the density perturbations for the case of quadratic inflation. Writing $V^{\prime \prime}(0)=\tilde{c} V(0) / M^{2}$ we now find

$$
\delta_{\mathrm{H}}^{2} \simeq \frac{V(0)^{3}}{75 \pi^{2} \tilde{c}^{2} V(0)^{2} \phi_{\mathrm{H}}^{2} M^{2}} .
$$

Note the appearance of the value, $\phi_{\mathrm{H}}$, of the (rescaled) inflaton field at the time of production of the observed density perturbations. Because of this, the scale of the inflationary potential now depends on $\phi_{\mathrm{H}}$ :

\footnotetext{
${ }^{2}$ The term 'chaotic' was in fact originally intended to refer to the initial conditions for the inflaton, with inflation occuring either at large [5] or small $\phi$ [6] field values. However it has come to be associated exclusively with large-field models with a generic monomial potential, which we discuss above. To avoid confusion we specifically refer to 'large-field' models where relevant. Note that when inflation occurs at small $\phi$ the initial conditions may alternatively be set by thermal effects [0]. This was originally found to be difficult to implement (hence the proposal of chaotic initial conditions (5]), but as we shall see these difficulties are circumvented if the inflationary scale is low.
} 


$$
V(\phi=0)^{1 / 4} \simeq 2 \times 10^{-2} \sqrt{\tilde{c}} \phi_{\mathrm{H}}^{1 / 2} M^{1 / 2}
$$

Thus if $\phi_{\mathrm{H}} / M$ is small, the scale of inflation will also be small even for natural values of $\tilde{c} \sim 1$. It is clear that quadratic inflation depends sensitively on the terms in the potential responsible for ending inflation and determining $\phi_{\mathrm{H}}$, and that if these terms generate a small value for $\phi_{\mathrm{H}} / M$ then we have a plausible mechanism for low-scale inflation. ? $^{3}$

Apart from being of quite general interest quadratic inflation has potential advantages for inflation in the case of models with new large dimensions. It has been shown that such models can evade the hierarchy problem associated with the existence of very large mass scales because in these models the Planck scale is no longer a fundamental quantity, instead all fundamental scales are of order the electroweak scale. In these models, however, inflation must be achieved via a potential which has no large scales and in this context the quadratic inflationary potential is necessary to generate acceptable slow-roll inflation. As we shall discuss models with new large dimensions also offer a new way of solving the $\eta$-problem.

The alternative explanation of the hierarchy problem is that a new symmetry - supersymmetry - is a good symmetry at low energy scales. As we have remarked earlier [8] supersymmetry provides a very natural source of viable inflatonary potentials. Supersymmetry prevents large radiative corrections to the potential and thus provides a consistent framework to address the $\eta$-problem. Furthermore, since thermal effects necessarily break supersymmetry, there is also a very natural explanation for why the inflaton field should have its initial high-temperature minimum far from its zero temperature supersymmetric minimum. In quadratic inflation models the scale of inflation can readily be identified with the scale of supersymmetry breaking.

In this paper we study the construction and implications of quadratic inflationary models, paying particular attention to the mechanisms for solving the $\eta$-problem, both in the context of supersymmetric models and in models with large new dimensions. In Section [1] we discuss the conditions that must be met by a quadratic inflaton potential in order to generate viable inflation. In Section IIII we introduce a simple parameterisation of a model capable of satisfying these conditions and study in detail in Section $\mathbb{I V}$ the characteristics of quadratic inflation. In Section $\nabla$ we discuss how the quadratic inflationary potential can arise in supergravity models and in Section $\nabla \mathbb{1}$ we discuss the same question in the context of theories with large new dimensions.

\section{QUADRATIC INFLATION}

Quadratic inflation requires that the inflaton field, $\phi$, rolls from the origin with an inflationary potential dominated by the quadratic term in a Taylor expansion. In field theory models such structure is quite a natural one because scalar fields often carry quantum numbers under a symmetry such that, in the limit where the symmetry is unbroken, a linear

\footnotetext{
${ }^{3}$ A potential with a leading quadratic term was in fact first studied in Ref. [6]. However it was the steepening of the potential due to this leading term that was assumed there to end inflation - this implies a relatively high value for $\phi_{\mathrm{H}}$, hence does not permit a low inflationary scale.
} 
term in the potential is forbidden and the quadratic term dominates. Of course it is necessary to show that the theory initially starts with the symmetry unbroken, i.e. $\phi=0$. We shall demonstrate that this can happen quite naturally through thermal effects because the dimensionless couplings of the theory necessarily respect the symmetry and give a thermal potential which has a minimum for vanishing $\phi$. It is also necessary that at low temperatures the inflaton starts to roll, spontaneously breaking the underlying symmetry. Again, as we shall discuss, this is quite natural as the quadratic mass term often has a negative sign, triggering spontaneous breaking of the symmetry. Provided this mass term is small it will not affect the high temperature potential significantly but will generate the slow-roll inflation at late times once the temperature drops sufficiently.

Of course this explanation requires that the system initially be in thermal equilibrium and this is not normally the case in slow-roll inflationary models, particularly since the inflaton should be very weakly coupled in order not to spoil the required flatness of its potential. However quadratic inflation is special in as much as the value of the potential during inflation is not strongly constrained by the need to generate the correct magnitude of density perturbations. For the case that the potential energy driving inflation is low, we will show that the processes leading to thermal equilibrium do have time to establish equilibrium before the inflationary era starts.

A further crucial question is why the universe should initially be sufficiently homogeneous for slow-roll inflation to begin. In chaotic (large-field) inflation models [5], inflation begins when the scale of the potential energy is of order the Planck scale and the horizon (the scale over which the universe must be homogeneous for inflation to start [9]) is also of order the Planck scale. By contrast in the models discussed here, inflation starts much later when the horizon contains many such Planck scale horizons and, in this case, it is difficult to understand how the necessary level of homogeneity can be realised. However this argument is not really a criticism of the possibility that there be a late stage of (quadratic) inflation but rather a statement that this cannot be all there is. Thus we require that there was some other process which ensured the necessary homogeneity at the beginning of quadratic inflation, as in the eternal inflation scenario [10], or perhaps through some quantum cosmological process [11. Such considerations suggest a situation in which a homogeneous universe emerges at the Planck era and potential energy is released reheating the universe and, as described above, setting the conditions for further periods of inflation to occur. If the late stage of inflation generates a sufficient number of e-folds of inflation, these earlier eras will not have observable consequences for our universe although they will have been crucial in setting the correct initial conditions for it to occur.

In a complete field theory description of the fundamental interactions there are usually many scalar fields, all candidates for generating a period of inflation. For this reason we consider quadratic inflation to be a generic feature. Indeed one might expect several inflationary periods to be encountered in the evolution of the universe due to several scalar fields slow-rolling to their minima. Again only the last such era will be relevant to observation if it generates a sufficiently large $(\sim 50-60)$ number of e-folds of inflation.

\footnotetext{
${ }^{4}$ Multiple short bursts of inflation may also be viable [12], as is indeed suggested by recent CMB and LSS data [13].
} 


\section{A. Slow-roll conditions}

Let us turn now to a more detailed discussion of the conditions that a quadratic potential must satisfy if it is to generate inflation. Starting from $\langle\phi\rangle \sim 0$ and assuming that the symmetry properties of the model forbid a linear term, the quadratic term will dominate, giving a new inflation [7] potential of the form?

$$
V(\phi) \sim \Delta^{4}-\frac{1}{2} m_{\phi}^{2} \phi^{2}+\ldots
$$

where the constant vacuum energy $\Delta^{4}$ is now the leading term in the potential.

The slow-roll condition is given by [2,14]

$$
\epsilon \equiv \frac{M^{2}}{2}\left(\frac{V^{\prime}}{V}\right)^{2} \ll 1, \quad|\eta| \equiv M^{2}\left|\frac{V^{\prime \prime}}{V}\right| \ll 1,
$$

where the potential determines the Hubble parameter during inflation as, $H_{\text {inf }} \equiv \dot{a} / a \simeq$ $\left(V / 3 M^{2}\right)^{1 / 2}$. Inflation ends (i.e. $\ddot{a}$, the acceleration of the cosmological scale factor, changes sign from positive to negative) when $\epsilon$ and/or $|\eta|$ becomes of $\mathcal{O}(1)$. From Eq.(8) we have now a constraint on the mass

$$
m_{\phi} \ll H_{\text {inf }} \sim \Delta^{2} / M
$$

which is much smaller than its natural value - the $\eta$-problem. To solve this problem we are driven to consider theories in which $m_{\phi}$ is prevented from becoming large.

\section{B. Initial conditions for inflation}

What about the initial conditions necessary for inflation to commence? We assume that some process at the Planck scale, presumably quantum cosmological in nature [11], creates a homogeneous patch of space-time of sufficient size for our last stage of inflation to occur if the inflaton field satisfies the constraints just discussed.

During the Planck era the inflaton field has a natural value of $\mathcal{O}\left(M_{\mathrm{P}}\right)$ due to gravitational interactions which are strong at this scale. At later times, while $\phi$ is still large, its potential will be dominated by the highest powers, $\phi^{p} / M^{\prime p-4}$, in the inflaton potential (where $M^{\prime}$ is a high mass scale in the theory). Such terms will cause the inflaton to flow towards the origin. However they cannot drive $\phi$ to a sufficiently small value for quadratic inflation to occur because they rapidly become negligible as $\phi$ becomes small. For this reason it is necessary to consider whether there exists some other mechanism capable of driving $\phi$ small.

\footnotetext{
${ }^{5}$ In what follows we will often just use $\phi$ to denote its vacuum expectation value (vev).

${ }^{6}$ In the case of additional dimensions this should presumably occur in the underlying higher dimensional theory at the higher dimensional Planck scale. The later stage of quadratic inflation occurs at a scale below the compactification scale when the effective theory is four-dimensional.
} 
In particular the thermal potential following from a coupling of the inflaton to other fields $\chi$ (e.g. $\phi^{2} \chi^{2}$ ) will contain a term proportional to $\phi^{2} T^{2}$ which can drive $\phi$ to the origin. Thus we should consider what happens after the Planck era to determine whether the necessary thermal distribution can be created from the potential energy released as the scalar fields, initially at the Planck scale, roll towards their low energy minima.

It is important to note that the thermalisation temperature cannot be close to the Planck scale, regardless of the amount of energy released. To quantify this let us consider the requirements on the inflaton field for it to be localised at the origin through its couplings to particles in the thermal bath [15]. On dimensional grounds, the $2 \rightarrow 2$ scattering/annihilation cross-sections at energies higher than the masses of the particles involved are expected to decrease with increasing temperature as $\sim \alpha^{2} / T^{2}$, where $\alpha$ is the coupling. Thus if the scattering/annihilation rate, $\Gamma \sim n\langle\sigma v\rangle$ is to exceed the Hubble expansion rate $H_{\text {therm }} \sim\left(g_{*} T^{4} / 10 M^{2}\right)^{1 / 2}$ in the radiation-dominated plasma, then we have a limit on the thermalisation temperature $T_{\text {therm }} \lesssim \alpha^{2} M / 3 g_{*}^{1 / 2}$, where $g_{*}$ counts the relativistic degrees of freedom $(=915 / 4$ in the minimal supersymmetric standard model (MSSM) at high temperatures). Now a coupling $g^{2} \phi^{2} \chi^{2}$ of the inflaton to another scalar field $\chi$ will generate a confining potential at high temperatures, $V(\phi, T) \sim g^{2} T^{2} \phi^{2}$ i.e. an effective mass for the inflaton of $m_{\text {therm }} \sim g T$. This will rapidly drive the inflaton field to the origin in a time of $\mathcal{O}\left(m_{\text {therm }}^{-1}\right)$. As the universe cools, the potential energy $\sim \Delta^{4}$ of the inflaton will begin to dominate over the thermal energy at a temperature $T_{\mathrm{inf}} \sim \Delta^{2} / \alpha^{2} M$. At this epoch the inflaton field will be localised to a region $\delta \phi \sim T_{\text {inf }}$ in the neighbourhood of the origin. Thus to provide natural initial conditions for quadratic inflation we require that:

$$
T_{\text {inf }}<T_{\text {therm }} \text { i.e. } \quad \Delta \lesssim 10^{-4} M \text { for } \quad \alpha \sim 1 / 24 .
$$

This rough estimate is consistent with the more precise explicit calculation of the $q \bar{q}$ annihilation rate into gluons which finds that equilibrium can only be attained below a temperature $T_{\text {therm }} \sim 3 \times 10^{14} \mathrm{GeV}$ [16. It is crucial if this mechanism is to work that the field $\chi$ should have a mass less than $T_{\text {therm }}$ otherwise it decouples and does not contribute to the thermal potential of the inflaton. This is a potential problem because the inflaton vev generates a mass $g \phi$. However, as we noted above, the term $\phi^{p} / M^{\prime p-4}$ does drive $\phi$ small, giving $g \phi=g M^{\prime}\left(T / M^{\prime}\right)^{4 / p}$. Thus a viable model capable of generating the initial conditions for inflation via thermal corrections has to satisfy the rather mild condition

$$
g M^{\prime}\left(T / M^{\prime}\right)^{4 / p}<T_{\text {therm }} .
$$

\section{ANALYSIS OF QUADRATIC INFLATIONARY MODELS}

In this Section we discuss the implications of quadratic inflation using a general parameterisation of the inflationary potential. The origin of this potential in specific theories will

\footnotetext{
${ }^{7} \mathrm{~A}$ similar estimate of the thermalisation temperature obtains in a study where cold particles are released at the Planck scale and allowed to scatter to achieve equilibrium 17 .
} 
be discussed in subsequent Sections. The potential can be conveniently parameterised as a constant term driving inflation plus a quadratic term with coefficient which may be a combination of a "bare" mass at the Planck scale together with a logarithmically varying mass term generated by radiative corrections [15]. Thus the full potential has the form

$$
V(\phi)=\Delta^{4}\left[1+\widetilde{b}\left(\frac{|\phi|}{M}\right)^{2}+\widetilde{c} \ln \left(\frac{|\phi|}{M}\right)\left(\frac{|\phi|}{M}\right)^{2}\right]
$$

Note that we have not included a term linear in $\phi$. As we have stressed above such a term can be forbidden if the theory has a symmetry under which $\phi$ transforms non-trivially and we assume that this is the case. For example $\phi$ may be a complex field which transforms under an Abelian symmetry (or a discrete subgroup) as $\phi \longrightarrow \mathrm{e}^{i \alpha} \phi$. In this case the lowest invariant we can form is $|\phi|^{2}$ as in Eq.(12) and $|\phi|$ is the component of $\phi$ that plays the role of the inflaton. In what follows we shall denote the inflaton simply by $\phi$.

In practice we are mainly interested in Eq.(12) for small $\phi \sim \phi_{\mathrm{H}}$, the field value when the density perturbations now entering our Hubble radius exit the horizon during inflation. In this region we can ignore, to a good approximation, the logarithmic variation of the effective mass term and write $V(\phi) \simeq \Delta^{4}\left(1+b \phi^{2}\right)$ where $b=\widetilde{b}+\widetilde{c} \ln \phi_{\mathrm{H}}^{2}$ and $\phi, \Delta$ are now expressed in Planck units: $\phi \equiv \phi / M, \Delta \equiv \Delta / M$. In this case we can solve the evolution equations analytically. After doing so we shall present numerical solutions to the potential including the logarithmically varying terms and compare with the analytic solution to the approximate form. Our parameterisation of the scaled potential $\bar{V}(\phi)=V(\phi) / \Delta^{4}$ is thus:

$$
\bar{V}(\phi)=\left(1-\frac{\kappa}{\Delta^{q}} \phi^{p}\right)^{2}+b \phi^{2}+c .
$$

For the case of interest, only the quadratic term is important during inflation. However to allow us to discuss the end of inflation we have included higher-order terms $\propto \phi^{p} / \Delta^{q}$ and above. These terms are only relevant to the behaviour at the end of inflation; motivation for this specific form is provided later (Section VC2). We have also included a constant, $c$ to allow the potential to vanish at the end of inflation. Note that a severe fine tuning in the value of $c$ is needed to cancel the contribution of the other terms at the true minimum of the potential. Since, to date, there is no explanation for the observed smallness of the cosmological constant, such a fine tuning is needed in any model of inflation and we are not able to improve on this situation. (However the required value of $c$ is so small that it plays no role in determining the nature of the inflationary era.)

\section{A. Analytical solution}

We first solve Eq.(13) quite generally, without requiring that the quadratic term dominates during inflation, although we will be most interested later in this particular case.

- The end of inflation: In the models under consideration inflation is generated while $\phi$ rolls to larger values. The end of inflation occurs at $\phi=\phi_{\mathrm{e}}$ when the slow roll conditions are violated. This occurs at $V^{\prime \prime}(\phi)=-\gamma$, where $\gamma \sim 1$. Thus we have 


$$
\phi_{\mathrm{e}} \approx\left[\frac{(\gamma+2 b) \Delta^{q}}{2 \kappa p(p-1)}\right]^{1 /(p-2)}
$$

- Scalar density perturbations: Solving the COBE normalisation equation

$$
\delta_{\mathrm{H}}^{2}(k)=\frac{1}{150 \pi^{2}} \frac{V_{\mathrm{H}}^{4}}{\epsilon_{\mathrm{H}}}
$$

we find

$$
\phi_{\mathrm{H}}^{p-1}-\frac{b \Delta^{q}}{\kappa p} \phi_{\mathrm{H}}-\frac{\Delta^{q+2}}{2 \kappa p A_{\mathrm{H}}}=0
$$

where $A_{\mathrm{H}} \equiv \sqrt{75} \pi \delta_{\mathrm{H}}$. This equation determines $\Delta$ once $\phi_{\mathrm{H}}$ is determined.

- Number of e-folds: The number of e-folds from $\phi_{\mathrm{H}}$ to the end of inflation at $\phi_{\mathrm{e}}$ is

$$
N_{\mathrm{H}} \equiv-\int_{\phi_{\mathrm{H}}}^{\phi_{\mathrm{e}}} \frac{V(\phi)}{V^{\prime}(\phi)} \mathrm{d} \phi \approx \int_{\phi_{\mathrm{H}}}^{\phi_{\mathrm{e}}} \mathrm{d} \phi \frac{1}{-2 b \phi+2 \kappa p \phi^{p-1} / \Delta^{q}}=\frac{1}{2 b(p-2)} \ln \left(\frac{1-b \Delta^{q} / \kappa p \phi_{\mathrm{e}}^{p-2}}{1-b \Delta^{q} / \kappa p \phi_{\mathrm{H}}^{p-2}}\right) .
$$

Solving for $\phi_{\mathrm{H}}$ gives

$$
\phi_{\mathrm{H}}=\left[\frac{b \Delta^{q}}{\kappa p\left\{1-\left(1-\frac{2 b(p-1)}{\gamma+2 b}\right) \mathrm{e}^{-2 b(p-2) N_{\mathrm{H}}}\right\}}\right]^{1 /(p-2)} \equiv B \Delta^{q /(p-2)} .
$$

Finally substituting in Eq.(16) and simplifying we obtain the required solution for $\Delta$ :

$$
\Delta=\left[2 \kappa p A_{\mathrm{H}}\left(B^{p-1}-\frac{b B}{\kappa p}\right)\right]^{p-2 /[2(p-2)-q]} .
$$

- Spectral Index: This is now easily obtained to be:

$$
\begin{aligned}
n_{\mathrm{H}} & \approx 1+2 V^{\prime \prime}\left(\phi_{\mathrm{H}}\right) \\
& \approx 1+4 b-4 \kappa p(p-1) B^{p-2} .
\end{aligned}
$$

\section{B. Numerical solution}

We now present numerical solutions for comparison with the analytical results obtained above. First we show in Fig. 1 the dependence of the inflationary scale $\Delta$ on the index $p$ for various values of $q$. The solid lines correspond to the analytical solution (19) showing how accurately these formulae reproduce the numerical results. Fig. 1 is obtained for negative values of $\widetilde{b}$ and spectral index $n_{\mathrm{H}}=0.9$ [18]. We observe that interesting scales for inflation are obtained in particular for the parameter sets $(p, q, \kappa)=(4,2,1),(4,3,1),(5,5,1)$ and $\left(5,5,1.8 \times 10^{-3}\right)$, as shown in Table $\mathbb{\square}$. In Fig. 2 we show the inflationary scale $\Delta$ as a function of the mass parameter $|b|$ to emphasise its insensitivity to the latter. 


\section{CHARACTERISTICS OF QUADRATIC INFLATION}

Using the form for the potential (13) it is straightforward to determine the properties of a wide variety of quadratic inflationary models characterised by the parameters $p, q$ and $\kappa$ which determine the end of inflation. In what follows we shall usually take $\kappa=1$, its "natural" value and discuss a few representative cases of the discrete possibilities specified by the integers $p$ and $q$. We shall also take the end of inflation to be set by $\gamma=1$, i.e. the epoch when the curvature of the potential becomes unity.

\section{A. The spectral index}

The value of $n_{\mathrm{H}}$ is shown as a function of the mass parameter $|b|$ in Fig. 3. One can see that a characteristic property of these models is that the spectral index is bounded as $n_{\mathrm{H}} \lesssim 0.95$ for the cases considered and that for large $|b|$ the index falls rapidly. One of the important issues is just how small $|b|$ must be to give acceptable inflation. This gives a measure of the severity of the $\eta$-problem and will allow us to determine whether the candidate explanations presented in Section VB do indeed solve the problem. Using the recent observational constraints on $n_{\mathrm{H}}$ [18] we can immediately obtain such a limit. In

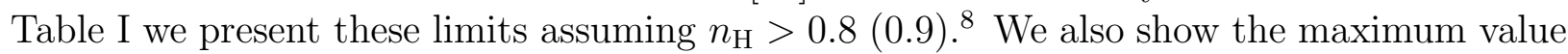
of the spectral index $n_{\mathrm{H} \text { max }}$ together with the corresponding $|b|_{\max }$ for which it is reached as seen in Fig. 3 .

\section{B. Fine tuning measure}

Examination of Table [ i shows that the maximum value of the effective mass squared parameter $b$ during inflation capable of generating density perturbations in the desired range is quite insensitive to the form of the potential at the end of inflation. As we discuss in Section VIB, in non-factorisable higher dimension models the value of $b$ is not constrained by the value of the inflationary potential so there is no fine tuning implied in this case. In supersymmetric models the "natural" value of $b$ is $\sim 1$ so we see that a fine tuning of about 1 part in 20 is required. In our opinion this is rather modest and can easily occur, particularly in models in which there are several candidate inflaton fields. As discussed in Section $\mathrm{VB}$, it can happen automatically in various supergravity schemes provided the required reduction in $b$ is relatively modest, namely $b \gtrsim h^{2} /(4 \pi)^{2}$ where $h$ is a coupling in the theory. We see that the required level of fine tuning lies comfortably in this range for reasonable choices of the coupling. We conclude therefore that it is quite likely that the necessary conditions for quadratic inflation will be realised in supergravity models. Indeed in realistic models there are usually a large number of fields associated with flat directions which are candidates for

\footnotetext{
${ }^{8}$ The recent Boomerang and MAXIMA observations of small angular-scale CMB anisotropy when combined with the COBE data indicate $n_{\mathrm{H}}=0.89 \pm 0.06$ if the baryon to photon ratio is fixed at the value indicated by nucleosynthesis arguments, and $n_{\mathrm{H}}=1.01_{-0.07}^{+0.09}$ otherwise [18]. The COBE data alone had previously indicated $n_{\mathrm{H}}=1.2 \pm 0.3$ 㭡.
} 
inflatons. In this case it will be the field with the flattest potential which will generate the last stage of inflation, the one relevant to our observable universe.

\section{Scale of inflation}

The scale, $\Delta$, of the inflationary potential during inflation is very sensitive to the form of the potential at the end of inflation. However since this form is determined by the discrete parameters $p$ and $q$, this should not be viewed as fine tuning - a given set will have a definite value for $\Delta$ (see Fig. 1). We can see from Table [ that a range of $\Delta$ from $1 \mathrm{GeV}$ upwards is obtained for reasonable choices of $p$ and $q$. The choice $\Delta \approx 10^{11} \mathrm{GeV}$ is particularly interesting since one can then identify $\Delta$ with the supersymmetry breaking scale in SUGRA models in which supersymmetry breaking is communicated from the hidden sector to the visible sector via gravitational strength couplings. As discussed in Section II B this value is low enough for thermal effects to set the initial value of the inflaton close to the origin, as is required if one is to have an inflationary era. Much lower values are possible, even down to $\sim 1 \mathrm{GeV}$ and these cases are relevant to the possibility of new large dimensions in which there is no fundamental scale much higher than the electroweak scale.

\section{The moduli problem}

Lowering the scale of the inflationary potential also provides a solution to the moduli problem. Moduli are scalar fields which, in the absence of symmetry breaking triggered by non-moduli fields, have no potential i.e. their vevs are undetermined. They are very common in string compactification e.g. the dilaton, the complex structure fields, and their vevs determine the gauge and Yukawa couplings of the theory. There are also moduli which determine the size and shape of the compactification manifold. Because the moduli have a very flat potential they suffer from a "moduli" problem due to the fact that during inflation the minimum of the moduli potential is typically at a different place from the minimum after inflation. Thus the moduli fields are trapped at a false minimum during inflation and this energy is released after inflation in the form of moduli excitations. If these excitations become non-relativistic before they decay into visible sector states they may release a large amount of entropy at a late stage, unacceptably diluting both baryonic and dark matter abundances. The reason the moduli problem may be evaded by lowering the scale of inflation is because the distorting effect of the inflation potential is proportional to its magnitude and so reduces as the fourth power of the inflation scale.

To make a quantitative estimate we follow our earlier detailed discussion [8]. First we note that the distorting contribution to the moduli potential is $M_{m}^{2} m^{2}$ where $M_{m}^{2} \lesssim V(0) / M_{\mathrm{P}}^{2}$; the inequality applies because, as noted for the inflaton, there are various possible ways the supersymmetry breaking mass scale can be reduced. This is generated by non-perturbative effects, such as supersymmetry breaking and may be characterised by $\Lambda^{4} f\left(m / M_{\mathrm{P}}\right)$, where $\Lambda$ is a symmetry breaking scale. This should now be compared to the inflaton potential after inflation. Consider first the case $\Lambda^{4}<V(0)$. In this case the moduli are lighter than the inflaton and thus decay after the reheating epoch. As discussed earlier [8] this leads

to an unacceptable release of entropy at late times. The problem can be neatly avoided if 
$\Lambda^{4}>V(0)$ for in this case the moduli decay harmlessly releasing their entropy before the inflaton decays.

What is the expectation for $\Lambda$ ? It has been pointed out [8] that for a large class of the moduli, $\Lambda$ may be identified with the scale of symmetry breaking (triggered by supersymmetry breaking effects) which occur below the string scale. In models with a large intermediate scale of breaking it is quite possible for $\Lambda^{4}$ to exceed $V(0)$, even in the case of linear inflation in which $V(0)$ is very large. However at least one modulus, the dilaton, is unaffected by such intermediate scale breaking and, as it is unlikely that it will get a mass much larger than the electroweak scale [19], it will be lighter than the inflaton in linear inflation models and will lead to an unacceptable late stage of entropy release. However in quadratic inflation the inflation scale can be very low. Indeed if $\sqrt{V(0)} / M_{\mathrm{P}}$ is less than the electroweak scale, the moduli problem may be solved even for the dilaton. We have seen in Section III that this is indeed possible.

So far our discussion has been in the context of four-dimensional space-time. If there are large new dimensions the moduli problem is even more severe because the moduli associated with the size and shape of the new dimensions cannot have mass greater than the fundamental higher dimensional scale of the theory, which may be close to the electroweak scale. The low inflation scale possible in quadratic inflation is essential in these models to avoid the moduli problem for these fields as well.

\section{Reheat temperature}

One obvious effect of lowering the scale of the inflationary potential is a decrease of the reheat temperature. At the end of inflation the field $\phi$ decays reheating the universe. The couplings of the inflaton to some other bosonic $\chi$ or fermionic $\psi$ MSSM fields occur due to terms $-\frac{1}{2} g^{2} \phi^{2} \chi^{2}$ or $-h \bar{\psi} \psi \phi$, respectively. These couplings induce decay rates of the form [1]

$$
\Gamma(\phi \rightarrow \chi \chi)=\frac{g^{4} \phi_{0}^{2}}{8 \pi m_{\phi}}, \quad \Gamma(\phi \rightarrow \bar{\psi} \psi)=\frac{h^{2} m_{\phi}}{8 \pi},
$$

where $\phi_{0} \approx\left(\Delta^{q} / \kappa\right)^{1 / p}$ is the value of $\phi$ at the minimum of the potential, and $m_{\phi}$ is the inflaton mass given by

$$
m_{\phi} \approx \sqrt{2} p \kappa^{1 / p} \Delta^{2-(q / p)}
$$

The decay rate is maximised when $m_{\chi, \psi} \sim m_{\phi}$ with $\Gamma \approx m_{\phi}^{3} / 8 \pi \phi_{0}^{2}$. The reheat temperature at the beginning of the radiation-dominated era is thus [14]

$T_{\text {reh }} \approx\left(\frac{90}{\pi^{2} g_{*}}\right)^{1 / 4} \min \left(\sqrt{H\left(\phi_{\mathrm{e}}\right) M}, \sqrt{\Gamma M}\right) \approx\left(\frac{30}{\pi^{2} g_{*}}\right)^{1 / 4} \min \left[\Delta,\left(\frac{3}{8 \pi^{2}}\right)^{1 / 4} p^{3 / 2} \kappa^{5 / 2 p} \Delta^{3-5 q / 2 p}\right]$.

The behaviour of $T_{\text {reh }}$ as a function of the mass parameter $|b|$ is shown in Fig. \&. Note that the observed baryon asymmetry of the universe may in principle be generated after reheating through anomalous electroweak $B+L$-violating processes and/or the Affleck-Dine mechanism, even for $T_{\text {reh }}$ as low as $\sim 1 \mathrm{GeV}$ [20]. 
So far we have explored the implications of quadratic inflation with a general parameterisation of the potential, without considering its specific origin. We turn now to a discussion of whether such potentials are reasonable in two attractive classes of models for physics beyond the Standard Model.

\section{SUPERGRAVITY INFLATION}

The only known symmetry capable of solving the hierarchy problem is supersymmetry which can guarantee that a scalar mass vanishes in the limit that supersymmetry is unbroken and ensures, through a cancellation of bosonic and fermionic contributions, that it is not affected by radiative corrections. However the non-vanishing potential of Eq.(7) driving inflation breaks (global) supersymmetry and so, even in supersymmetric models, all scalar masses during inflation are non-zero in general. In the extreme case that the inflaton has vanishing non-gravitational couplings, gravitational effects will typically induce a mass of order $H_{\text {inf }} \sim \Delta^{2} / M$ for any scalar field [21], in particular the inflaton [22]. Nevertheless this is a big improvement over the non-supersymmetric case, for the fine tuning problem now simply becomes one of requiring $m_{\phi}=\beta \Delta^{2} / M$ with $\beta \lesssim 0.1$ to obtain successful inflation. In this Section we briefly review supergravity models which can lead to this form and discuss the structure of the resulting inflaton potential.

\section{A. The Supergravity potential}

In $N=1$ supersymmetric theories with a single SUSY generator, complex scalar fields are the lowest components, $\phi^{a}$, of chiral superfields, $\Phi^{a}$, which contain chiral fermions, $\psi^{a}$, as their other components. In what follows we will take $\Phi^{a}$ to be left-handed chiral superfields so that $\psi^{a}$ are left-handed massless fermions. Masses for fields will be generated by spontaneous symmetry breakdown so that the only fundamental mass scale is the normalised Planck scale. This is aesthetically attractive and is also what follows if the underlying theory generating the effective low-energy supergravity theory emerges from the superstring. The $N=1$ supergravity theory describing the interaction of the chiral superfields is specified by the Kähler potential [23],

$$
G\left(\Phi, \Phi^{\dagger}\right)=d\left(\Phi, \Phi^{\dagger}\right)+\ln |f(\Phi)|^{2}
$$

Here $d$ and $f$ (the superpotential) are two functions which need to be specified; they must be chosen to be invariant under the symmetries of the theory. The dimension of $d$ is 2 and that of $f$ is 3 , so terms bilinear (trilinear) in the superfields appear without any mass factors in $d(f)$. The scalar potential following from Eq.(24) is given by [23]

$$
V=\mathrm{e}^{d / M^{2}}\left[F^{A \dagger}\left(d_{A}^{B}\right)^{-1} F_{B}-3 \frac{|f|^{2}}{M^{2}}\right]+D-\text { terms }
$$

where

$$
F_{A} \equiv \frac{\partial f}{\partial \Phi^{A}}+\left(\frac{\partial d}{\partial \Phi^{A}}\right) \frac{f}{M^{2}}, \quad\left(d_{A}^{B}\right)^{-1} \equiv\left(\frac{\partial^{2} d}{\partial \Phi^{A} \partial \Phi_{B}^{\dagger}}\right)^{-1}
$$


At any point in the space of scalar fields $\Phi$ we can make a combination of a Kähler transformation and a holomorphic field redefinition such that $\phi^{a}=0$ at that point and the Kähler potential takes the form $d=\sum_{a}\left|\Phi_{a}\right|^{2}+\ldots$ In this form, the scalar kinetic terms are canonical at $\phi^{a}=0$ and from Eq. 25), neglecting $D$-terms and simplifying to the case of a single scalar field, the scalar potential coming from the $F$-terms has the form

$$
V_{F}=\left(\mathrm{e}^{|\phi|^{2} / M^{2}+\ldots}\right)\left[\left|\left(f_{\phi}+f \phi^{*}+\ldots\right)(1+\ldots)\right|^{2}-3 \frac{|f|^{2}}{M^{2}}\right]=V_{0}+|\phi|^{2} \frac{V_{0}}{M^{2}}+\ldots,
$$

where $\left.V_{0} \equiv V_{F}\right|_{\phi=0}$.

\section{B. Supersymmetry breaking and the $\eta$ problem}

During inflation supersymmetry is broken by the non-zero inflaton potential, $V$. For the case of $F$-term inflation $V_{0} \neq 0$ and we see from Eq.(27) that the resultant breaking of supersymmetry gives all scalar fields a contribution to their mass-squared of $V_{0} / M^{2}$. If this is the only contribution to the mass there is an obvious conflict with the slow-roll condition (8) on $\eta$. This is the essential problem one must solve if one is to implement inflation in a supergravity theory. However, as stressed earlier, the problem is relatively mild when compared to the non-supersymmetric case because the suppression for $\eta$ need only be by a factor of 10 or so.

There have been several proposals for dealing with this problem. One widely explored possibility is $D$-term inflation [24]. In particular one may consider an anomalous $D$-term in Eq.(25) of the form

$$
V_{D}=\frac{g^{2}}{2}\left(\xi-\sum_{i} q_{i}\left|\widetilde{\phi}_{i}\right|^{2}\right)^{2}
$$

where $\xi$ is a constant and $\widetilde{\phi}_{i}$ are scalar fields charged under the anomalous $U(1)$ with charge $q_{i}$. Such a term, for vanishing $\widetilde{\phi}_{i}$, gives a constant term in the potential but, unlike the case of $F$-term inflation, $V_{D}$ does not contribute in leading order to the masses of uncharged scalar fields. The latter occur in radiative order only giving a contribution to their masssquared equal to $\beta^{2} V_{0} / M^{2}$ where $\beta^{2} \approx h^{\prime 2} / 16 \pi^{2}$, and typically $\beta \approx 10^{-1}$ for an effective coupling $h^{\prime} \sim 1$ between the charged and neutral fields.

The other suggested ways out of the $\eta$ problem do not require non-zero $D$-terms during inflation but rather provide reasons why the quadratic term in Eq.(27) should be anomalously small. One possibility follows from the fact that the mass-squared term of $V_{0} / M^{2}$ coming from Eq.(27) actually applies at the Planck scale. Since we are considering inflation for field values near the origin the inflaton mass-squared must be run down to low scales. As shown in Ref. [25], in a wide class of models in which the gauge couplings become large at the Planck scale the low energy supersymmetry breaking soft masses are driven much smaller

at low scales by radiative corrections. The typical effect is to reduce the mass by a factor of $\alpha(M) / \alpha(\mu)$ where $\alpha(\mu)$ is a gauge coupling evaluated at the scale $\mu$. While radiative corrections can cause a significant change in the coupling, the effect is limited and becomes smaller as the gauge coupling becomes small. For this reason the effective mass at low scales cannot be arbitrarily small and typically $\beta \gtrsim 1 / 25$. 
It is also possible to construct models in which the contribution to the scalar mass exhibited in Eq.(27) is cancelled by further contributions coming from the expansion of $f$ in Eq.(25). One interesting example which arises in specific superstring theories was discussed in Ref. [26]. Another occurs in supergravity models of the 'no-scale' type [27]. In these examples, while the scalar mass is absent in leading order, it typically arises in radiative order and so again there is an expectation that the effective mass cannot be arbitrarily small.

To summarise, for small inflaton field values, in all these cases the effective supergravity potential can be conveniently parameterised as a constant term driving inflation plus a quadratic term with coefficient which may be a combination of a "bare" mass at the Planck scale together with a logarithmically varying mass term generated by radiative corrections. Thus the full potential has just the form (12) discussed earlier.

\section{Terminating slow-roll evolution}

So far we have concentrated on the form of the potential which arises due to supersymmetry breaking effects which inevitably follow from the existence of a non-zero value for the potential during inflation. However one may expect additional terms in the potential which are allowed even when supersymmetry is unbroken. These terms will determine the position of the minimum of the potential after inflation and may also be responsible for ending inflation.

It is this latter possibility that we shall concentrate on here because the nature of the inflationary era is largely determined by when inflation ends. The reason the end of inflation is likely to be triggered by additional terms in the potential follows from a simple dimensional argument. In the models we are considering, the inflaton starts with field value close to the origin and rolls to larger field values. Close to the origin the quadratic terms are likely to dominate but as the inflaton field value increases, higher-order terms in $\phi / M$ are likely to dominate. Such terms may occur through higher-order terms in $d$ or $f$ in Eq.(24) or in higher-order terms in the expansion of Eq.(27). The form of these terms is restricted by any symmetries (discrete or continuous) of the theory. In compactified string theories such symmetries abound, determined by the shape of the compactification manifold. Consider first the form of the potential following from the superpotential $f$. In a string theory, in the absence of symmetry breaking, the superpotential is cubic in the superfields. In the effective low energy theory below the string and compactification scales, higher-order terms may arise as a result of integrating out heavy modes. The resulting form of the superpotential depends on how the inflaton transforms under the symmetries of the theory. It is not possible to give an exhaustive list of all possible symmetries so here we will consider simple examples to illustrate the possibilities.

\section{Symmetries of the inflaton potential}

We start with the case where the inflaton is a singlet under all continuous gauge symmetries but transforms under a $Z_{q}$ discrete symmetry (which may be a discrete gauge symmetry and thus protected from large gravitational corrections [28]). The leading term in the super-

potential is then of the form $\phi^{q} / M^{q-3}$ where we have taken the mass scale associated with 
this term to be the reduced Planck mass, $M$. (This scale arises as a result of integrating out heavy degrees of freedom associated with the string scale or the compactification scale so here we are taking all such scales to be of $\mathcal{O}(M)$.) Following from Eq.(27) we see that this term gives rise to the leading terms of the form $|\phi|^{2 q-2} / M^{2 q-6}$ in the scalar potential. Note that this term is not suppressed by the factor $\Delta^{4} / M^{2}$ associated with the quadratic $|\phi|^{2}$ term because it arises even in the absence of supersymmetry breaking. This has the important effect of reducing the value of $\bar{\phi} / M$ at which the term $|\phi|^{2 q-2} / M^{2 q-6}$ becomes larger than the quadratic term. We see from the slow roll conditions (8) that shortly thereafter this term will cause inflation to end. The effect of these higher-order terms may be enhanced still further if they arise as a result of integrating out heavy modes lighter than the Planck scale. For example the superpotential $f=\phi^{r} X / M^{r-2}+M_{X} X^{2}$ describes the interaction of $\phi$ with a new field $X$ which has mass $M_{X}$. The form of this superpotential follows from a $Z_{2 r}$ symmetry under which $\phi$ and $X$ have charge 1 and $r$ respectively. At scales below $M_{X}$ the field $X$ may be integrated out to give the effective superpotential $f=\phi^{2 r} /\left(M^{2 r-4} M_{X}\right)$. Now the scale of the higher dimension operator is set by a combination of $M$ and $M_{X}$ and for $M_{X}<M$ will enhance the contribution of the higher dimension term. The new mass $M_{X}$ could be any of the scales in the theory, e.g. the inflationary scale or the supersymmetry breaking scale, and can thus be much smaller than the Planck scale.

\section{A simple parameterisation}

With this preamble we now consider how to parameterise the structure of the terms responsible for ending inflation. Consider first the inflationary models driven by a non-zero $F$-term. This is conveniently parameterised by the superpotential $f=\Delta^{2} Y$ which gives $V=\left|F_{Y}\right|^{2}=\Delta^{4}$ as required. Radiative corrections then lead to the form of Eq.(12). As discussed above we expect higher-order corrections involving the inflaton to appear and there are many possible forms for such corrections depending on the underlying symmetries of the theory. Here we present a convenient parameterisation of the potential which follows from a simple symmetry to demonstrate how a complete inflation potential may be driven by the symmetry structure. The starting point is the superpotential $f=\Delta^{2} Y$. Such a form linear in $Y$ follows if $Y$ carries non-zero $R$-symmetry charge $2 \beta$ under an unbroken $R$-symmetry because the full superpotential must also have charge $2 \beta$ under such a symmetry. Now let $\phi$ be a singlet under the $R$-symmetry but have a charge under a discrete $Z_{p}$ symmetry. Then the most general superpotential has the form

$$
f=\left(\Delta^{2}-\frac{\phi^{p}}{M^{\prime p-2}}-\frac{\phi^{2 p}}{M^{2 p-2}}-\ldots\right) Y,
$$

where we have suppressed the coefficients of $\mathcal{O}(1)$ of each term. This gives rise to the potential

$$
V=\left(\Delta^{2}-\frac{\phi^{p}}{M^{\prime p-2}}-\frac{\phi^{2 p}}{M^{\prime 2 p-2}}-\ldots\right)^{2},
$$

plus terms involving $Y$ which we drop as they do not contribute to the vacuum energy (since $Y$ does not acquire a vacuum expectation value). For small $\phi$ the leading term has 
the form $\Delta^{2} \phi^{p} / M^{\prime p-2}$ and, for the purpose of ending inflation, this is all that matters the higher-order terms in Eq.(30) do not play a role during inflation. Thus this form of the superpotential gives the same inflationary phenomenology as discussed in the previous subsection with the superpotential $\phi^{q} / M^{q-3}$, provided the leading higher-order terms in $\phi$ are the same, i.e. $p=2 q-2$ and $M=M^{\prime}\left(M^{\prime} / \Delta\right)^{1 /(q-3)}$. This illustrates the more general point that different symmetries may lead to the same inflationary potential. For our parameterisation we use a slightly simplified form of Eq.(30) keeping only the leading $\phi^{p}$ term and setting $M^{p-2}=\Delta^{q-2} M^{p-q}$ to take account of the possibility discussed above that the scale associated with the higher dimension operators may be below the Planck scale. Note $q$ is an integer as the term $\Delta^{q}$ comes from heavy propagators when integrating out massive fields. Thus we arrive at the form

$$
V=\Delta^{4}\left(1-\kappa \frac{\phi^{p}}{\Delta^{q} M^{p-q}}\right)^{2}+\Delta^{4}\left[\widetilde{b}\left(\frac{|\phi|}{M}\right)^{2}+\widetilde{c} \ln \left(\frac{|\phi|}{M}\right)\left(\frac{|\phi|}{M}\right)^{2}\right]
$$

where we have added the supersymmetry breaking terms of Eq.(12). As discussd earlier, this form of the inflationary potential allows for a variety of inflationary scales $\Delta$ depending on the choice of $p$ and $q$ and our results for the potential (13) in Table $[$ should apply with the interpretation $b=\widetilde{b}+\widetilde{c} \ln \phi_{\mathrm{H}}^{2}$. In Table $\mathbb{\Pi}$ we show the results of a numerical integration of the potential (31), demonstrating that this is indeed the case. To conclude this discussion we show in detail how the symmetries lead to the higher-order term $\phi^{p} / M^{p-2}=\Delta^{q-2} M^{p-q}$ in the superpotential for three representative cases, identifying the origin of the mass scale $M^{\prime}$ and hence the source of the $\Delta^{q}$ factor.

- Case 1: $p=4, q=2, \Delta \approx 10^{11} \mathrm{GeV}$

This simple case follows immediately from a $Z_{4}$ symmetry under which $\phi$ has unit charge and $Y$ is neutral. Taken together with the $R$-symmetry the leading term allowed is $Y \phi^{4} / M^{2}$ where $M$ is the fundamental mass scale of the theory which we take to be the Planck scale. Combined with the supersymmetry breaking term $Y \Delta^{2}$ we immediately obtain Eq.(31) with $p=4, q=2$. As discussed above, this potential gives acceptable density perturbations provided $\Delta \approx 10^{11} \mathrm{GeV}$, i.e. it can be identified with the supersymmetry breaking scale. Fig. 5 shows the full supergravity inflaton potential (25) as a function of $\phi$ and its phase $\alpha$ for $(p, q, \kappa)=(4,2,1)$.

- Case 2: $p=4, q=3, \Delta \approx 10^{5} \mathrm{GeV}$

In this case we choose the superpotential of the form

$$
f=Y \phi X+M_{X} X \bar{X}+\bar{X} \phi^{3} / M
$$

This form arises if there is a $U(1)$ (or $Z_{n}$ ) symmetry under which the fields $Y, X, \bar{X}, \phi$ have charge $-4,3,-3$ and 1 respectively. Integrating out the massive $X, \bar{X}$ fields gives

$$
f=Y \phi^{4} /\left(M M_{X}\right) \text {. }
$$

If $M_{X}=\Delta$ (as is expected if $X, \bar{X}$ belong to the supersymmetry breaking sector driven by a gaugino condensate in which the (confining) interactions become strong 
at the scale $\Delta$ ) we have the desired term in the superpotential which, when combined with the supersymmetry breaking term $Y \Delta^{2}$ (the SUSY breaking condensate, $\Delta^{2}$, also breaks the $U(1)$ (or $Z_{n}$ ) symmetry), yields the form given in Eq.(31).

- Case 3: $p=5, q=5, \Delta \approx 1 \mathrm{GeV}$

This is readily achieved along similar lines to the previous case via the superpotential

$$
f=Y \phi^{2} Z+\bar{Z} X^{2}+\bar{X} \phi^{2}+M_{Z} Z \bar{Z}+M_{X} X \bar{X}
$$

This form arises if there is a $U(1)$ (or $Z_{n}$ ) symmetry under which the fields $Y, Z, \bar{Z}, X, \bar{X}, \phi$ have charge $-10,8,-8,4,-4$ and 2 respectively. Integrating out the massive $X, \bar{X}$ fields gives

$$
f=Y \phi^{5} / M_{X}^{2} M_{Z}
$$

With $M_{X}=M_{Z}=\Delta$ and the supersymmetry breaking term $Y \Delta^{2}$ we arrive at a superpotential giving Eq.(31) with $p=5, q=5$. Fig. 6, similar to Fig. 5, but now for $(p, q, \kappa)=(5,5,1)$, shows how lower scales of inflation require lower values of $\phi$; in particular we see how the minimum $\phi_{0}$ shifts to smaller values.

\section{D-term inflation}

So far we have discussed the form of the inflaton potential responsible for ending inflation that may arise as a result of $F$-term inflation. However it is possible to obtain similar forms using $D$-term inflation and, as noted in Section $\nabla \mathrm{B}$, this may have the advantage of eliminating the $\eta$ problem. At first sight it seems impossible to obtain $D$-term inflation with a low scale for the inflationary potential because the usual assumption is that the non-zero value of the $D$-term is due to an anomalous $U(1)$ as in Eq.(28) and its scale is in turn related to the string scale. However there are two possible ways that a low $D$-term scale may arise. The first possibility is that the string scale itself is low. With the realisation that the fourdimensional Planck scale may not be a fundamental quantity has come the construction of string models with low string scales (even as low as $\sim 1 \mathrm{TeV}$ ) plus large new dimensions. In these theories the weakness of the gravitational interactions is due to the gravitational flux spreading out in new large extra space dimensions or through the appearance of a warp factor in the 4-D metric dependent on the additional dimensions. Either way the string scale and hence the associated anomalous $D$-term is reduced. The second possibility applies even in the original string formulations with small extra dimensions. The fields in the hidden supersymmetry breaking sector all feel the strong confining force responsible for gaugino condensation. Such fields may be driven to acquire vevs of the order of the scale $\Delta$ at which the force becomes strong. These vevs may give rise to non-zero $D$-terms capable of generating inflation at the scale $\Delta$. Given these possibilities what is the form of the potential and does it have similar properties to that of Eq.(31)? In both of the cases just discussed the general form of the $D$-term is given by

$$
V_{D}=\left.|| \Delta\right|^{2}-X^{\dagger} X+\left.\bar{X}^{\dagger} \bar{X}\right|^{2},
$$


where we have included fields $X$ and $\bar{X}$ carrying +1 and -1 charge respectively under the $U(1)$ gauge symmetry. The end of $D$-term inflation must be driven in a different manner from that discussed above, namely through hybrid inflation. During inflation the $X$ field is prevented from acquiring a vev because it has a mass, $M_{X}$, which we take to come from the supersymmetry breaking sector and hence to be of $\mathcal{O}(\Delta)$. The end of inflation corresponds to the point at which the change in the vev of the inflaton, $\phi$, which is a singlet under the gauge symmetry associated with the non-zero $D$-term, must alter the potential of $X$ in such a way that it can acquire a vev cancelling the $D$-term. The most general form of the superpotential is (suppressing Yukawa couplings)

$$
f=M_{X} X \bar{X}+\phi X \bar{X}+\text { higher }- \text { order terms }
$$

The potential following from this includes the leading terms

$$
V_{F}=\left|M_{X}+\phi\right|^{2}\left(|X|^{2}+|\bar{X}|^{2}\right)+|X \bar{X}|^{2}
$$

For $\phi \approx 0$ the $X$ field has mass $M_{X}$ and for $M_{X} \gtrsim \Delta$ the potential $V_{D}+V_{F}$ will constrain $X$ to have zero vev. Once the vev of $\phi$ becomes of $\mathcal{O}\left(M_{X}\right)$ however a cancellation of the $X$ mass term is possible and the $X$ field will rapidly evolve so as to minimise $V_{D}$. Since the $X$ mass scale is $\gtrsim \Delta$ this happens within a Hubble time and so inflation is effectively ended. In fact for $M_{X}$ of $\mathcal{O}(\Delta)$ this reproduces Case 1 discussed above because there too the end of inflation occurred at $\phi$ of $\mathcal{O}(\Delta)$. Variations on this hybrid theme can readily generate the other cases too. Replacing the $\phi \bar{X}$ term in Eq.(37) by the term $\phi^{s} X \bar{X} / \Delta^{t}$ (such a term can be obtained by integrating out fields in an analogous way to that discussed above) one finds inflation ends for $\phi=\left(\Delta^{t} M_{X}\right)^{1 / s}$. For the potential of the form given in Eq. (31) the end of inflation occurs at $\phi \approx \Delta^{q /(p-2)}$ and so for $M_{X}$ of $\mathcal{O}(\Delta)$ and $q /(p-2)=(t+1) / s$, the end of inflation will be the same in the $D$-term case.

\section{4-D INFLATION IN HIGHER DIMENSION THEORIES}

As mentioned above there has recently been much interest in a solution to the hierarchy problem involving $\delta$ new large dimensions in which gravity (described by closed strings in a string theory) propagate in the $4+\delta$ dimensions while the matter states of our world, quarks, leptons and the gauge bosons responsible for the strong, weak and electromagnetic interactions, (described by open string states whose ends are confined to D-branes) live in just the normal four-dimensional space (the $\mathrm{D}=3$ case). The description of inflation in more than 4 dimensions may be done either by using the full higher dimensional description or by using an effective 4-D description in which the effects of the higher dimension appear as towers of Kaluza Klein states. Which description is more appropriate depends on the energy scale of interest. Here we use the four-dimensional description because it is very likely that during inflation the effective temperature drops below the compactification scale at which the extra dimensions are frozen. Even if inflation starts at a scale above the compactification scale for the additional dimensions, during inflation the universe cools to the Hawking temperature $\sim \sqrt{V(0)} / M$, and, if this is below the compactification scale, the theory will be effectively four-dimensional during inflation. If this is not the case we expect 
problems because the phase transition corresponding to compactification will occur after inflation. While this is not necessarily "no-go", it is rather disfavoured since the moduli setting the scale of the new dimensions propagate in the additional dimensions and are likely to produce an unacceptable amount of entropy in the bulk.

In the effective 4-D description, it is necessary to discuss how the presence of additional large dimensions may change the form of the slow-roll equations.

\section{A. Factorisable metric}

In the original realisation of this idea 229] one assumes a factorisable metric of the form

$$
\mathrm{d} s^{2}=-\mathrm{d} x^{0} \mathrm{~d} x_{0}+\mathrm{d} x_{i} \mathrm{~d} x^{i}+\mathrm{d} x_{\alpha} \mathrm{d} x^{\alpha}, \quad i=1,2,3, \quad \alpha=4, \ldots, 4+\delta,
$$

and finds that, due to the gravitational flux leaking out into the extra dimensions, the gravitational coupling to matter in $4-\mathrm{D}$ is much weaker than in the higher dimensional space, with the 4-D Planck mass given by

$$
M_{\mathrm{P}, 4}^{2}=M_{\mathrm{P}, 4+\delta}^{\delta+2} R^{\delta}
$$

Thus provided $M_{\mathrm{P}, 4+\delta} R \gg 1$, one can explain why the four-dimensional Planck mass is large while the fundamental mass scale $M_{\mathrm{P}, 4+\delta}$ remains small. In the extreme one may take $M_{\mathrm{P}, 4+\delta}$ of $\mathcal{O}(1) \mathrm{TeV}$ ), i.e. small enough to eliminate the hierarchy problem which occurs when one introduces scales much larger than the electroweak scale. In such theories there is no mass scale higher than $M_{\mathrm{P}, 4+\delta}$. Moreover the reheat temperature must be extremely low [29] if the bulk is not to contain so much energy that it distorts the expansion rate of the universe in an unacceptable way. Given this it is clear that the only viable inflationary model is one which does not require a high scale for the inflaton potential, pointing at quadratic inflation as the obvious candidate. Moreover it is necessary for the theory still to be supersymmetric in order to explain why the inflaton mass is only of order the Hubble expansion parameter during inflation (i.e. the inflation sector supersymmetry breaking scale) — without supersymmetry one expects it would be driven to the scale $M_{\mathrm{P}, 4+\delta}$. Thus the discussion about the form of the inflation potential in supersymmetric theories applies to factorisable metric compactifications as well. The important difference is that in Eq.(31) $M$ should be identified with $M_{\mathrm{P}, 4+\delta}$ and not with the 4-D Planck mass. At first sight this does not seem to be the correct prescription because the inflaton, a brane state, has only gravitational strength couplings $\propto M_{\mathrm{P}, 4}^{-n}$ to the new Kaluza Klein states responsible for the additional dimensions in our 4-D description. However after summing over all the Kaluza Klein states these couplings do induce higher-order corrections $\propto \phi^{n+4} / M_{\mathrm{P}, 4+\delta}^{n}$.

\section{B. Non-factorisable metric}

Recently there has been renewed interest in the case where the metric is not factorisable. For the case of a single extra dimension the line element has the form

$$
\mathrm{d} s^{2}=\mathrm{e}^{-\rho(r)}\left(-\mathrm{d} x^{0} \mathrm{~d} x_{0}+\mathrm{d} x_{i} \mathrm{~d} x^{i}\right)+\mathrm{d} x_{4} \mathrm{~d} x^{4}, \quad i=1,2,3,
$$


where $x_{4}=r \theta, 0<\theta \leq 2 \pi$. The origin of the hierarchy between the electroweak breaking scale and the Planck scale is now rather different than that suggested for the factorisable metric case. An explicit example was recently provided by Randall and Sundrum [30]. They considered the case of two parallel 3-branes sitting on the fixed points of an $S^{1} / Z_{2}$ orbifold. The 5-D spacetime is essentially a slice of five-dimensional Ant-DeSitter spacetime and the tensions of the two 3-branes are chosen so that the 4-D spacetime appears flat. This last requirement forces one of the two branes to have negative tension. The solution to Einstein's equations now has the form of Eq.(41) with $\rho(r)=k R$ where $k$ is the five-dimensional curvature. The exponential "warp" factor, $\varpi=\mathrm{e}^{-k R}$, in the metric then generates a hierarchy of the mass scales between the two branes, although all fundamental mass scales are of order $M_{\mathrm{P}, 4}$. The graviton is localised to the positive tension brane while matter exists on the negative tension brane with particle masses of order $\varpi M_{\mathrm{P}, 4}$. Due to the exponential dependence of the warp factor on the size of the new dimension, one may readily generate the desired mass hierarchy with $\varpi \sim 10^{-1}$ even though the size, $R$, of the orbifold is only $\sim 30$ times $k^{-1}$ which is of order the Planck length.

It may seem that such schemes have a great advantage in generating slow-roll inflation by easily solving the $\eta$-problem. This follows because these models provide a reason why the inflaton mass should be less than the Hubble parameter during inflation even without supersymmetry. The reason is that, due to the universal warp factor, all masses on a given brane involve the warp factor suppression. As we have just discussed this is why on the visible brane all masses are of order the electroweak breaking scale. If the inflaton lives on the visible brane (or a brane very close to it) it too will have an electroweak scale mass which is stable against radiative corrections from sectors on other branes. In this scheme there should be vacuum energy, $V(0)$, (the net contribution from the bulk and the branes) driving inflation until the inflaton rolls to its minimum to cancel the vacuum energy. Unlike the factorisable case there is no constraint on the fundamental higher-dimensional scale. As a result $V(0)$ can be large, even as large as the 4-D Planck scale. Thus, in this case, both linear and quadratic inflation appear viable even without supersymmetry.

However, if $V(0)$ is much greater than the scale, $\Lambda$, on the inflaton brane, the inflaton will not be able to cancel $V(0)$ fully. This is because to cancel $V(0)$ the inflaton vev must be of order $V(0)^{1 / 4}$. However above the scale $\Lambda$ on the inflaton brane the underlying higherdimension theory (string theory?) must be used because the effective theory has uncontrolled higher-dimension terms of the form $\phi^{n} / \Lambda^{n-4}$. Thus even in this case we need a mechanism to ensure that the inflaton is anomalously light to satisfy the slow-roll equations (8). The obvious candidate is supersymmetry as already discussed.

Up to now this discussion has been at a qualitative level. To be more explicit we consider the five-dimensional case in which the Standard Model states and the inflaton live on a 3brane while gravity propagates in the full five dimensions. We start with the 5-D description. Solution of the Einstein equations for the case that the metric is projected on to a spatially flat Friedmann-Robertson-Walker (FRW) model and the 4-D cosmological constant after inflation is set to zero gives a modified form for the Hubble expansion rate [32 34]

$$
H^{2}=\frac{8 \pi}{3 M_{4}^{2}} \rho\left(1+\frac{\rho}{2 \lambda}\right),
$$

where $\lambda$ is the brane tension, $\rho$ is the energy density and we have used $M_{4}$ to denote $M_{\mathrm{P}, 4}$. It is given by 


$$
M_{4}=\sqrt{\frac{3}{4 \pi}}\left(\frac{M_{5}^{2}}{\sqrt{\lambda}}\right) M_{5}
$$

where $M_{5}=M_{\mathrm{P}, 5}$. In Eq.(42) the term quadratic in $\rho$ corrects the standard FRW cosmology. However the effective 4-D Lagrangian should have just the Einstein-Hilbert form and one might expect to recover the standard cosmology. In fact this is the case, provided the Planck constant appearing therein is time dependent. P It is easy to see this during inflation when $\rho=V(\phi)$. Expanding the potential about the point where density perturbations leave the horizon we find

$$
H^{2}=\frac{1}{3 M_{4}^{2}} V(0)\left(1+\frac{V(0)}{2 \lambda}\right)\left(1+V^{\prime}(0) \phi+\frac{1}{2} V^{\prime \prime}(0) \phi^{2}+\ldots\right),
$$

where we have dropped terms of $\mathcal{O}(\phi / \lambda)$. One can see that this has just the normal 4-D form but with a modified Planck mass

$$
M_{4}^{\prime 2}=M_{4}^{2}\left(1+\frac{V(0)}{2 \lambda}\right)^{-1}
$$

The case of chaotic (large-field) inflation (i.e. linear inflation in our terminology) has been explored in Ref. [35]. In this case, for $M_{5} \ll M_{4}$, the new term quadratic in $V$ in Eq. (42) is large, so the modified Planck mass is reduced with

$$
M_{4}^{\prime 2} \simeq 10^{4} M_{5}^{2}
$$

As a result, following the discussion leading to Eq.(3), we see that linear inflation generates the correct magnitude of density perturbations with the scale

$$
V(0)^{1 / 4} \simeq 2 \sqrt{c} M_{5}
$$

which can be low for $M_{5}$ low. Indeed in the chaotic inflationary model investigated [35] the scale of inflation is $\sim M_{5} / 10$. This demonstrates a new mechanism for generating low-scale inflation without requiring quadratic inflation or supersymmetry, which is apparently in conflict with the general arguments given above!

To explain this we note that Eq.(44) relies on the variation of the Planck scale between the period perturbations are produced and today, the variation corresponding to the variation of the horizon size. This will not be the case for compact extra dimensions [30] if inflation occurs after the size of the extra dimensions are fixed because the horizon then is bigger than the compactification radius. As argued above, we consider this to be the likely case if the bulk is to remain empty. In this case the Planck mass does not differ during and after inflation and, following the arguments above, one sees that linear inflation requires the scale of inflation to be as large as in Eq.(3). As a result, if one is to have a low inflationary scale consistent with a low fundamental scale $M_{5}$ on the brane, it is necessary to consider quadratic inflation. Moreover, as discussed above, it is then also necessary to invoke supersymmetry to

\footnotetext{
${ }^{9}$ We are grateful to A. Karch for helpful comments concerning this point.
} 
keep the coefficient of the quadratic term below the Hubble rate as required by the slow-roll conditions (8).

For the case that the additional dimensions are infinite [31] the result of Eq. (45) is a possibility, the variation of the Planck scale corresponding to the variation of the horizon. The new ingredient is that there is no new compact dimension and furthermore the Planck scale is time-dependent. However even in this case there are reasons why one needs supersymmetric quadratic inflation. We note that the specific chaotic inflation model of ref. 355 requires an inflaton mass $m \simeq 5 \times 10^{-5} M_{5}$ and thus there must be supersymmetry or some other mechanism responsible to keep the inflaton mass much less than its natural scale $M_{5}$, as noted earlier [36]. Moreover even this is not sufficient to make linear low-scale inflation viable. The value of the inflaton vev at the time perturbations are produced is $\phi^{\prime} \sim 3 \times 10^{2} M_{5}$. Since non-renormalisable quantum corrections occur at $\mathcal{O}\left(\phi^{\prime n+4} / M_{5}^{n}\right)$, such large values of the inflaton field are likely to destroy the flatness of the potential required for inflation. Indeed, as discussed above, for $\phi^{\prime}$ above $M_{5}$ it is necessary to use the underlying (string?) theory which is needed to regulate the $\mathcal{O}\left(\phi^{\prime n+4} / M_{5}^{n}\right)$ terms. For the case of quadratic inflation these problems need not arise because the scale of the inflationary potential, $V(0)$, can be much smaller than the brane tension $\lambda$ so, c.f. Eq.(42), the form of the inflationary potential at the time the observable perturbations are produced is just the normal 4-D one analysed in Section $\mathbb{1 1}$.

\section{SUMMARY}

In this paper we have explored the possibility that there could be an inflationary era associated with a low scale for the inflationary potential. The most extensively studied models correspond to the case where the inflaton potential is linear in a Taylor expansion about the value of the inflaton field just before the time when the observable density perturbations are produced. In these models the scale of inflation is restricted to lie close to the Planck scale by the requirement that scalar density perturbations should have their observed magnitude. On the other hand, models in which the inflaton potential is quadratic have density perturbations which depend sensitively on the value of the inflaton field at the end of inflation. We have explored the implications of such models in detail and found they can generate acceptable perturbations even if the scale of inflation is the electroweak scale or even lower.

Low scale inflation has several attractive features. It offers a solution to the troublesome moduli problem associated with superstring compactification. At least in the context of supersymmetric theories, thermal effects readily impose the required initial conditions needed for such inflation to occur. If there are large new space dimensions solving the hierarchy problem, a low scale of inflation is essential as there are no large fundamental mass scales.

The construction of a viable inflationary model requires an explanation as to why the inflaton potential should be much flatter than dimensional arguments using the fundamental mass scale of the theory would suggest. The most promising solution is if there is an underlying supersymmetry which maintains the flatness of the inflaton potential even in the presence of a large (supersymmetry breaking) cosmological (near) constant term. Simple supersymmetric models readily lead, through large non-renormalisable terms, to the rapid end of inflation needed to achieve low scale inflation. We have also considered the possibility for low scale inflation in models with large new dimensions. Although these models offer 
an alternative non-supersymmetric explanation for the hierarchy problem they still require an additional mechanism to keep the inflaton potential flat, again suggesting the need for supersymmetry.

Determining the nature of the underlying theory leading to inflation is a difficult task. The forthcoming precision CMB and LSS measurements will certainly play an important role in this and we have seen that low scale quadratic inflationary models give a characteristic prediction ('tilted' or 'red') for the spectral index of the scalar density perturbation. Laboratory experiments will play a complementary role because the implication of an underlying supersymmetry is that there should be new supersymmetric states which are likely to be observable in the next generation of experiments. Thus there is a good chance that the ideas investigated in this paper may be tested within the next decade.

\section{ACKNOWLEDGMENTS}

GG gratefully acknowledges financial support from the Royal Society and the Mexican Academy of Sciences, which made possible a visit to the University of Oxford; he also acknowledges support by UNAM project PAPIIT IN110200. GGR would like to thank the Aspen Institute of Physics, where part of this work was undertaken (supported by TMR

grant FMRX-CT96-0090) and P. Binetruy, A. Karch, R. Maartens and L. Randall for useful discussions. 


\section{TABLES}

\begin{tabular}{|c|c|c|c|c|c|c|l|l|}
\hline \hline$p$ & $q$ & $\kappa$ & $n_{\mathrm{H}}$ & $n_{\mathrm{H}, \max }$ & $|b|$ & $|b|_{\max }$ & $\Delta(\mathrm{GeV})$ & $\Delta_{\max }(\mathrm{GeV})$ \\
\hline 4 & 2 & 1 & $0.8(0.9)$ & 0.95 & $0.05(0.024)$ & 0.005 & $7(46) \times 10^{10}$ & $50 \times 10^{10}$ \\
4 & 3 & 1 & $0.8(0.9)$ & 0.93 & $0.05(0.024)$ & 0.007 & $4.6(27) \times 10^{4}$ & $28.7 \times 10^{4}$ \\
5 & 5 & 1 & $0.8(0.9)$ & 0.92 & $0.05(0.024)$ & 0.01 & $1.3(4.2)$ & 2.9 \\
5 & 5 & $1.5 \times 10^{-3}$ & $0.8(0.9)$ & 0.94 & $0.05(0.024)$ & 0.008 & $1(10) \times 10^{2}$ & $7.6 \times 10^{2}$ \\
\hline \hline
\end{tabular}

TABLE I. Characteristics of representative quadratic inflation models for the potential (13). The values of $|b|$ and $\Delta$ correspond to the assumed condition $n_{\mathrm{H}}>0.8(0.9)$.

\begin{tabular}{|c|c|c|c|c|c|}
\hline$p$ & $q$ & $\kappa$ & $n_{\mathrm{H}}$ & $|b|$ & $\Delta(\mathrm{GeV})$ \\
\hline 4 & 2 & 1 & $0.8(0.9)$ & $0.05(0.026)$ & $1(3.7) \times 10^{11}$ \\
\hline 4 & 3 & 1 & $0.8(0.9)$ & $0.05(0.026)$ & $5.4(22.4) \times 10^{4}$ \\
\hline 5 & 5 & 1 & $0.8(0.9)$ & $0.05(0.025)$ & $1.3(3.6)$ \\
\hline 5 & 5 & $1.5 \times 10^{-3}$ & 0.9 & 0.026 & $10^{3}$ \\
\hline
\end{tabular}

TABLE II. Characteristics of representative quadratic inflation models for the potential (31) where we have chosen $\widetilde{b}=25 \widetilde{c}$. For comparison with Table $\left[\right.$ we give the value of $b=\widetilde{b}+\widetilde{c} \ln \phi_{\mathrm{H}}^{2}$, the effective mass at the epoch when the fluctuations observed by COBE leave the horizon. 


\section{FIGURES}

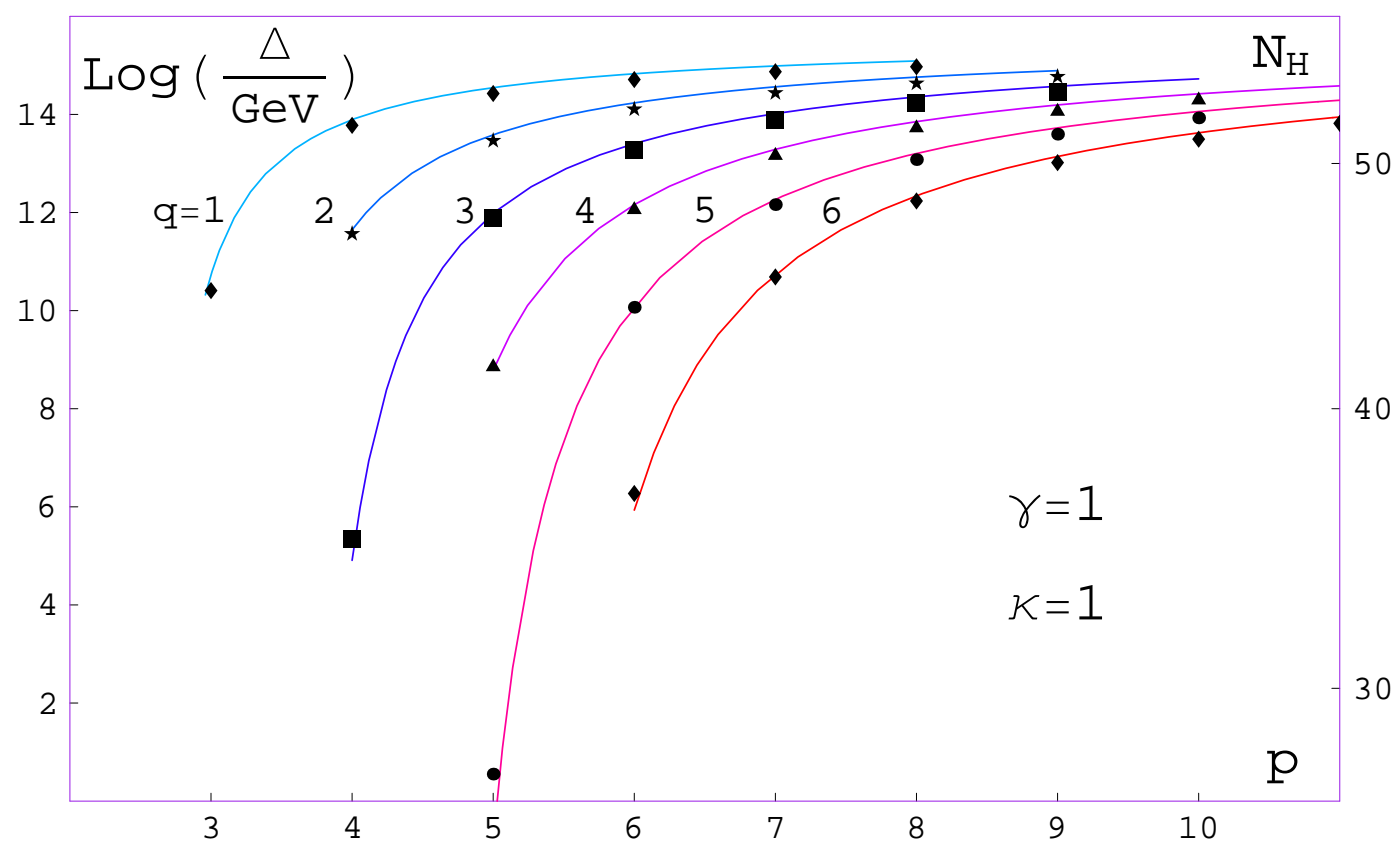

FIG. 1. The logarithm of the inflationary scale $\Delta$ as a function of $p$ for various values of $q$. The solid lines are the analytical solution (19) while the symbols indicate the numerical results, obtained assuming $\widetilde{b}$ to be negative and spectral index $n_{\mathrm{H}}=0.9$. Interesting scales of inflation correspond in particular to the set of values $(p, q, \kappa)=(4,2,1),(4,3,1)$ and $(5,5,1)$, as shown in the Tables. The number of e-folds $N_{\mathrm{H}}$ indicated on the right-hand axis is an approximation.

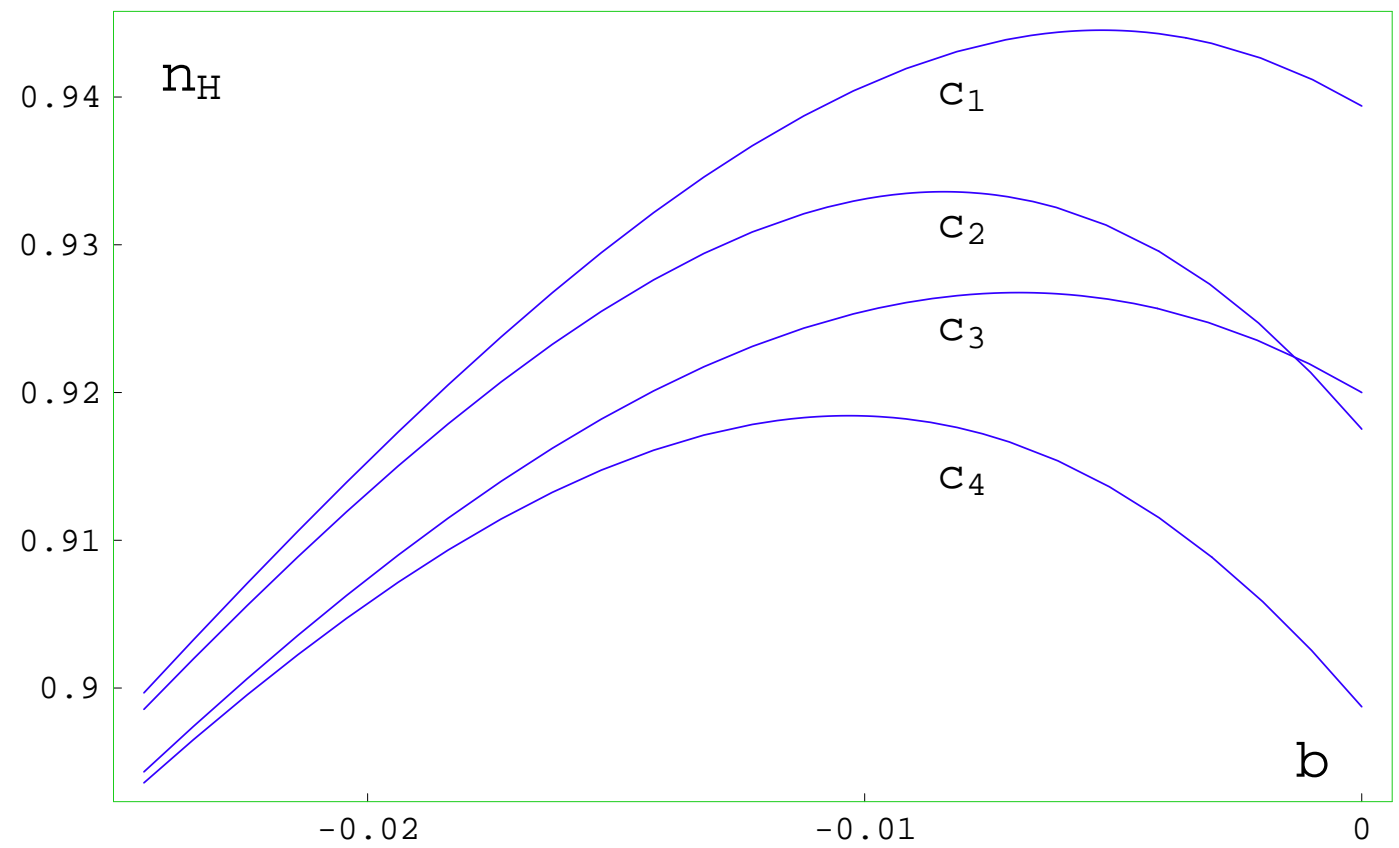

FIG. 2. The spectral index $n_{\mathrm{H}}(20)$ as a function of the mass parameter $b$ for the cases $(p, q, \kappa)=(4,2,1),(4,3,1),\left(5,5,1.8 \times 10^{-3}\right)$ and $(5,5,1)$, denoted by $c_{1}, c_{2}, c_{3}$ and $c_{4}$ respectively. 


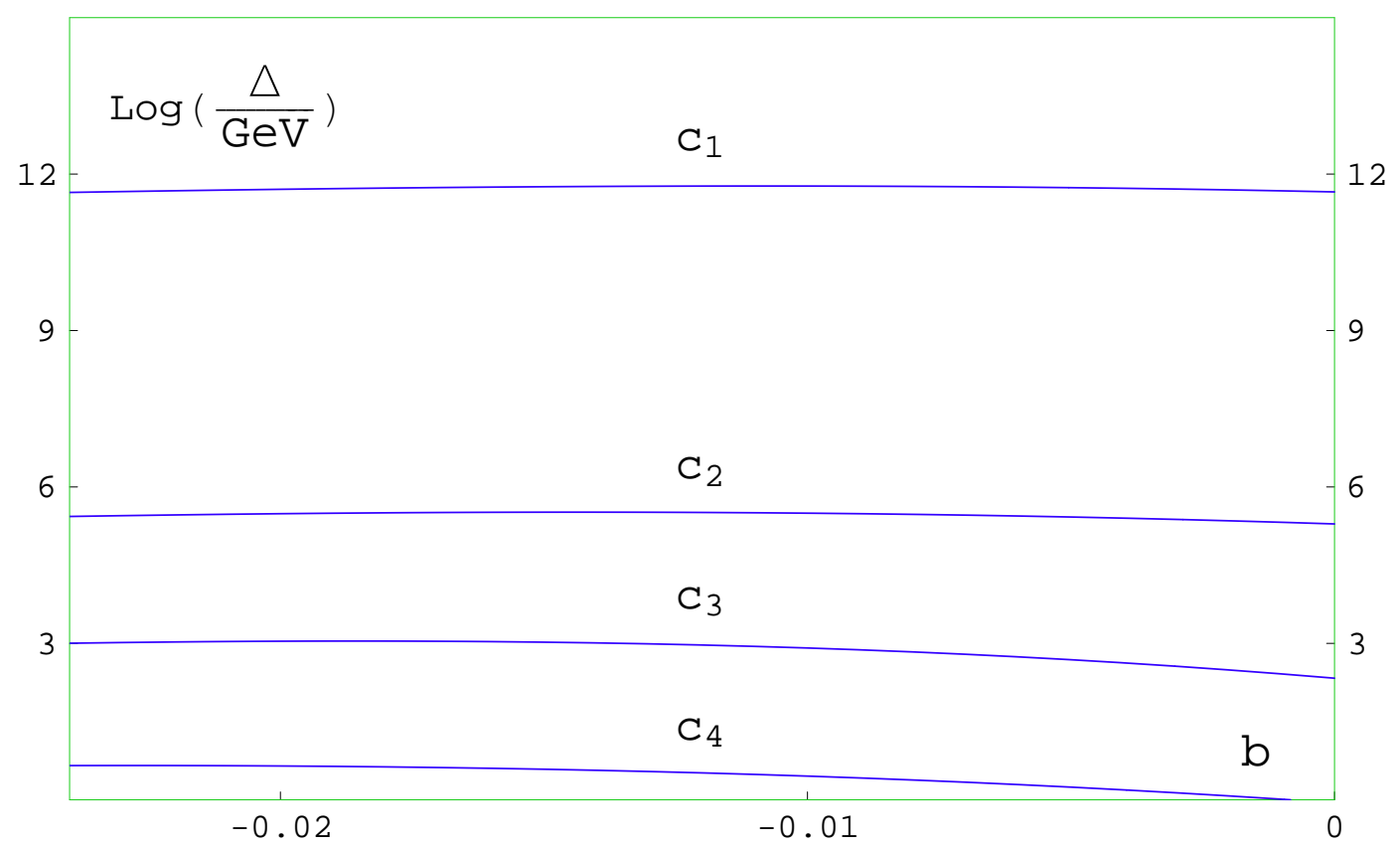

FIG. 3. The logarithm of the inflationary scale $\Delta(19)$ as a function of the mass parameter $b$ for the same cases as in Fig. 2.

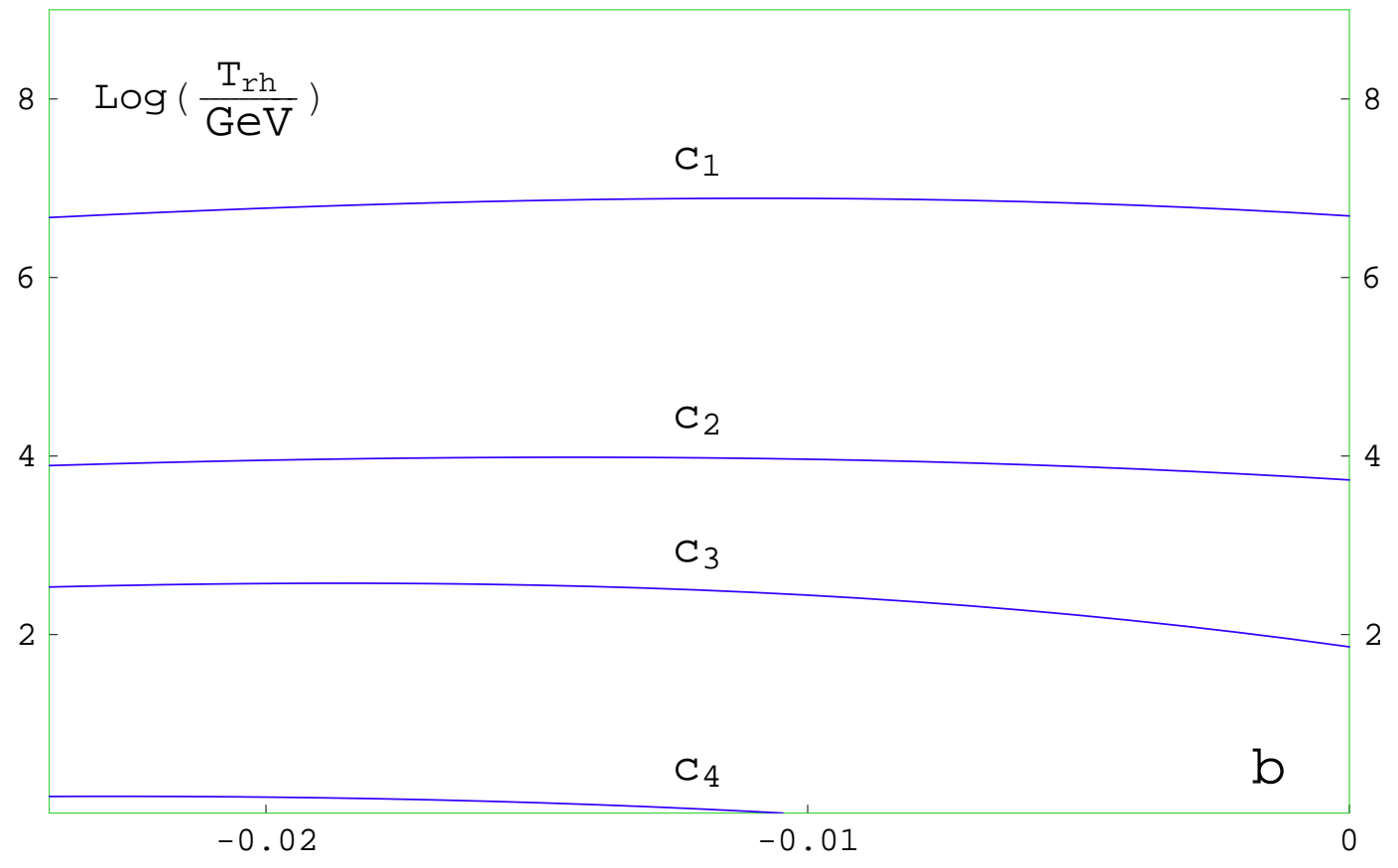

FIG. 4. The logarithm of the reheat temperature $T_{\text {reh }}(23)$ as a function of the mass parameter $b$ for the same cases as in Fig. 2. 


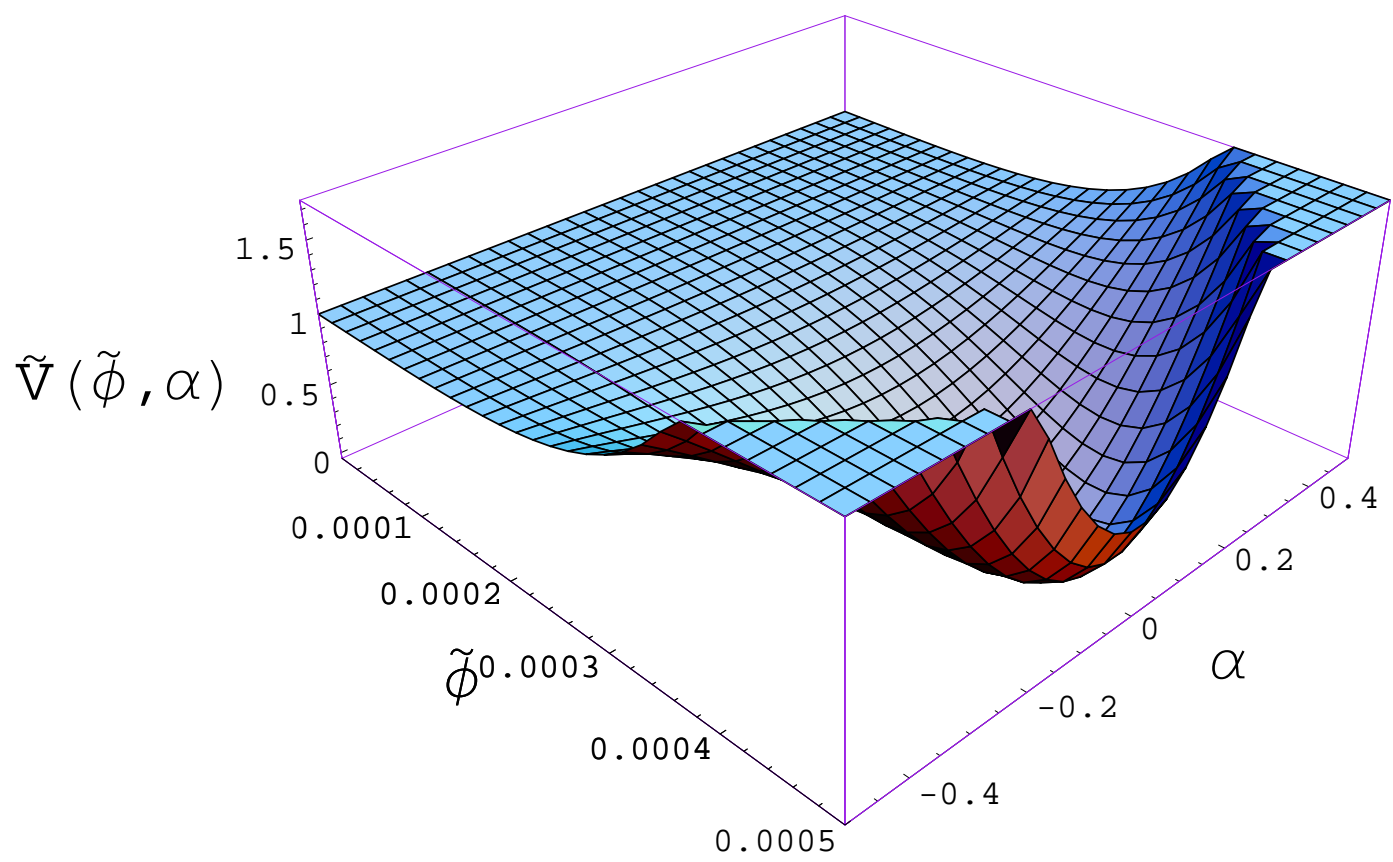

FIG. 5. The full supergravity potential (25) (in units of $V_{0} / \Delta^{4}$ ) as a function of $\phi$ and its phase $\alpha$ for the case $(p, q, \kappa)=(4,2,1)$, corresponding to an inflationary scale of $\Delta \sim 5 \times 10^{11} \mathrm{GeV}$.

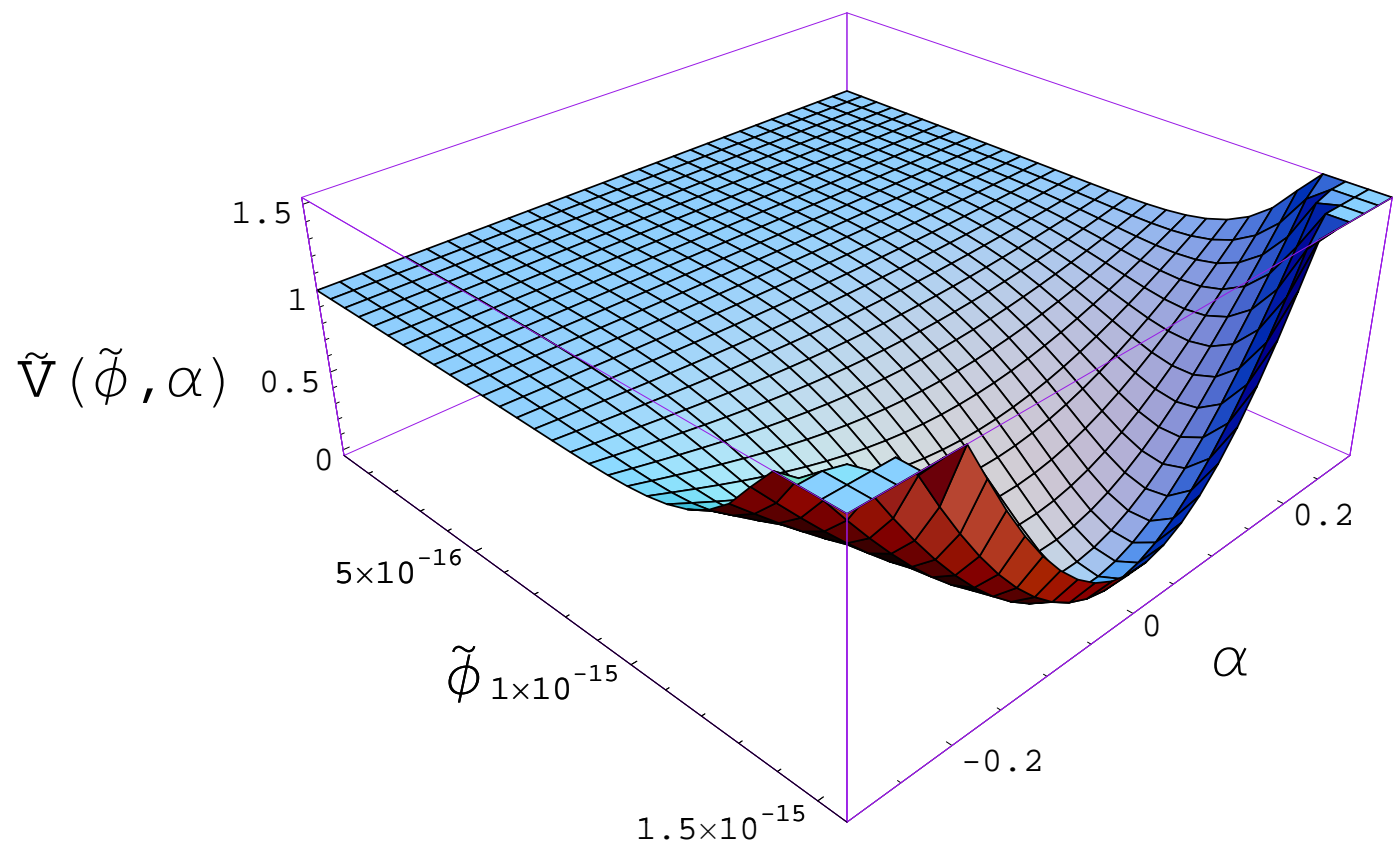

FIG. 6. Similar to Fig. 0 , but for the case $(p, q, \kappa)=(5,5,1)$ corresponding to an inflationary scale of $\Delta \sim 1 \mathrm{GeV}$. 


\section{REFERENCES}

[1] A.D. Linde, Particle Physics and Inflationary Cosmology (Harwood Academic, 1990); E.W. Kolb and M.S. Turner, The Early Universe (Addison-Wesley, 1990).

[2] D. Lyth, A. Riotto, Phys. Rep. 314 (1999) 1.

[3] J.E. Lidsey, A.R. Liddle, E.W. Kolb, E.J. Copeland, T. Barreiro, M. Abney, Rev. Mod. Phys. 69 (1997) 373

[4] C.L. Bennett et al, Astrophys. J. 464 (1996) L1;

E.F. Bunn, M. White, Astrophys. J. 486 (1997) 6.

[5] A.D. Linde, Phys. Lett. 129B (1983) 177.

[6] A.D. Linde, Phys. Lett. 132B (1983) 317.

[7] A.D. Linde, Phys. Lett. 108B (1982) 389;

A. Albrecht, P.J. Steinhardt, Phys. Rev. Lett. 48 (1982) 1220.

[8] G.G. Ross, S. Sarkar, Nucl. Phys. B461 (1996) 597.

[9] T. Vachaspati, M. Trodden, Phys. Rev. D61 (2000) 023502;

A. Berera, C. Gordon, Phys. Rev. 63 (2001) 063505.

[10] P.J. Steinhardt, in The Very Early Universe, eds G.W. Gibbons, S.W. Hawking, S. Siklos (Cambridge University Press, 1982), p. 251;

A.D. Linde, Nonsingular Regenerating Inflationary Universe, Cambridge University Report Print-82-0554 (1982);

A. Vilenkin, Phys. Rev. D27 (1983) 2848;

A. Guth and S.-Y. Pi, Phys. Rev. D32 (1985) 1899;

A. Linde, D. Linde, A. Mezhlumian, Phys. Rev. D49 (1994) 1783;

A. Linde and D. Linde, Phys. Rev. D50 (1994) 2456.

[11] S.W. Hawking, N. Turok, Phys. Lett. B425 (1998) 25;

A. Vilenkin, Phys. Rev. D57 (1998) 7069;

A.D. Linde, Phys. Rev. D58 (1998) 083514;

N. Turok, S.W. Hawking, Phys. Lett. B432 (1998) 271;

For a review, see, N. Turok, astro-ph/0011195.

[12] J.A. Adams, G.G. Ross, S. Sarkar, Nucl. Phys. B503 (1997) 405.

[13] J. Barriga, E. Gaztañaga, M.G. Santos, S. Sarkar, Mon. Not. R. Astr. Soc., to appear (astro-ph/0011398).

[14] P.J. Steinhardt, M.S. Turner, Phys. Rev. D29 (1984) 2162.

[15] G. Germán, G.G. Ross, S. Sarkar, Phys. Lett. 469B (1999) 46.

[16] K. Enqvist, J. Sirkka, Phys. Lett. B314 (1993) 298;

[17] K. Enqvist, K.J. Eskola, Mod. Phys. Lett. A5 (1990) 1919.

[18] A.H. Jaffe et al, Phys. Rev. Lett. 86 (2001) 3475.

[19] T. Banks, M. Berkooz, P.J. Steinhardt, Phys. Rev. D52 (1995) 705;

T. Banks et al, Phys. Rev. D52 (1995) 3548.

[20] see, e.g., S. Davidson, M. Losada, A. Riotto, Phys. Rev. Lett. 84 (2000) 4284.

[21] M. Dine, W. Fischler, D. Nemechansky, Phys. Lett. 136B (1984) 169;

G. Coughlan, W. Fischler, E.W. Kolb, S. Raby, G.G. Ross, Phys. Lett. 140B (1984) 44.

[22] E. Copeland, A.R. Liddle, D.H. Lyth, E.D. Stewart, D. Wands, Phys. Rev. D49 (1994) 6410.

[23] D. Bailin, A. Love, Supersymmetric Gauge Field Theory and String Theory (Adam Hilger, 1994). 
[24] E.D. Stewart, Phys. Rev. D51 (1995) 6847;

E. Halyo, Phys. Lett. B387 (1996) 43;

P. Binétruy, G. Dvali, Phys. Lett. B388 (1996) 241;

For a review and further references, see Ref. [2].

[25] S.F. King, G.G. Ross, Nucl. Phys. B530 (1998) 3.

[26] J.A. Casas, G.B. Gelmini, Phys. Lett. B410 (1997) 36;

J.A. Casas, G.B. Gelmini, A. Riotto, Phys. Lett. B459 (1999) 91.

[27] M.K. Gaillard, H. Murayama, K.A. Olive, Phys. Lett. B355 (1995) 71;

M. Bastero-Gil, S.F. King, Nucl.Phys. B549 (1999) 391.

[28] L.E. Ibanez, G.G. Ross, Nucl. Phys. B368 (1992) 3, Phys. Lett. B260 (1991) 291.

[29] N. Arkani-Hamed, S. Dimopoulos, G. Dvali, Phys. Lett. B429 (1998) 263.

[30] L. Randall, R. Sundrum, Phys. Rev. Lett. 83 (1999) 3370.

[31] L. Randall, R. Sundrum, Phys. Rev. Lett. 83 (1999) 4690.

[32] T. Shiromizu, K. Maeda, M. Sasaki, Phys. Rev. D62 (2000) 024012;

P. Binetruy, C. Deffayet, U. Ellwanger, D. Langlois, Phys. Lett. B477 (2000) 285.

[33] P. Kanti, I. Kogan, K. Olive, M. Pospelov, Phys. Lett. B468 (1999) 31, Phys. Rev D61 (2000) 106004;

T. Nihei, Phys. Lett. B465 (1999) 81;

N. Kaloper, Phys. Rev. D60 (1999) 123506;

C. Csaki, M. Graesser, C. Kolda, J. Terning, Phys. Lett. B462 (1999) 34;

J.M. Cline, C. Grojean, G. Servant, Phys. Rev. Lett. 83 (1999) 4245.

[34] H.B. Kim, H.D. Kim, Phys. Rev. D61 (2000) 064003;

E.E. Flanagan, S.H. Tye, I. Wasserman, Phys. Rev. D62 (2000) 044039.

[35] R. Maartens, D. Wands, B. Bassett, I. Heard, Phys. Rev. D62 (2000) 041301.

[36] D.H. Lyth, Phys. Lett. B448 (1999) 191. 